\title{
OPTICAL DESIGN OF WFIRST-AFTA WIDE-FIELD INSTRUMENT
}

\author{
Bert Pasquale \\ NASA Goddard Space Flight Center \\ David Content, Jeffrey Kruk, David Vaughn, \\ Qian Gong, Joseph Howard, Alden Jurling, \\ Eric Mentzell, Nerses Armani; \\ NASA Goddard Space Flight Center \\ Gary Kuan, Jet Propulsion Laboratory
}




\section{W]}

\section{WFIST-AFTA Optical Design}

- Wide-Field Infra-Red Survey Telescope Astrophysics Focused Telescope Assets

- Makes use of unused telescope components from the intelligence community, repurposed to NASA for scientific use.

- Optical Design: 2.36-meter aperture Three Mirror Anastigmat (TMA)

- Constrained and influenced by a combination of existing hardware, design heritage, science interests and volume constraints 


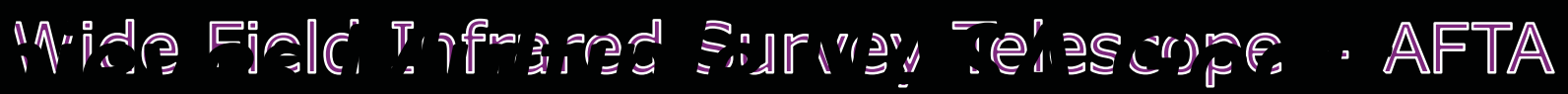

Hits 5 of 6 NASA Strategic Goals

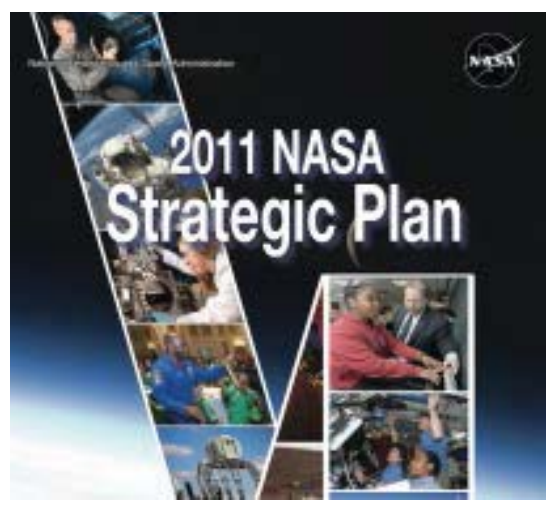

\#1 Large Mission Priority WFIRST science

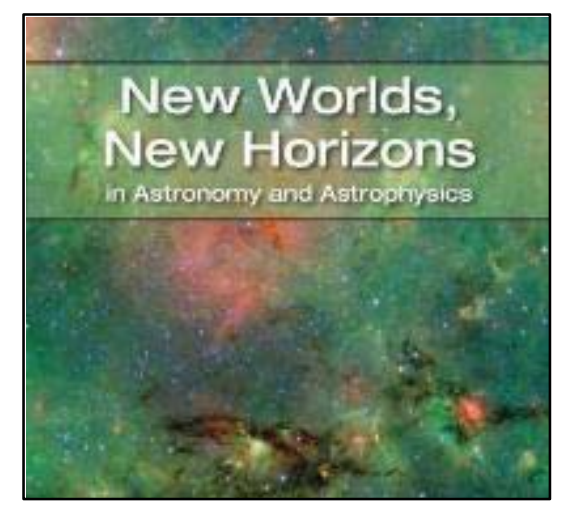

\#1 Medium Scale Priority Exoplanet Imaging

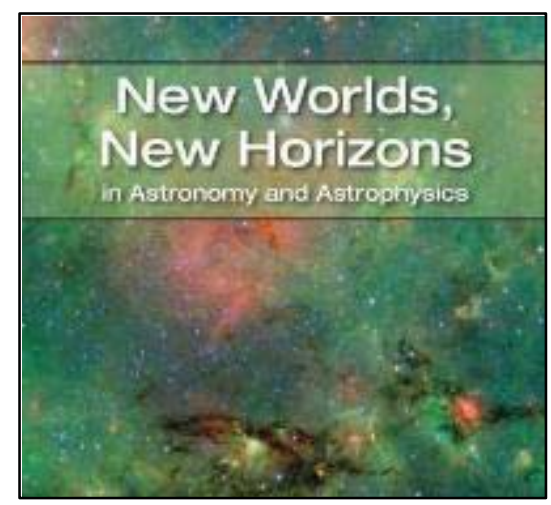

Nobel Prize science

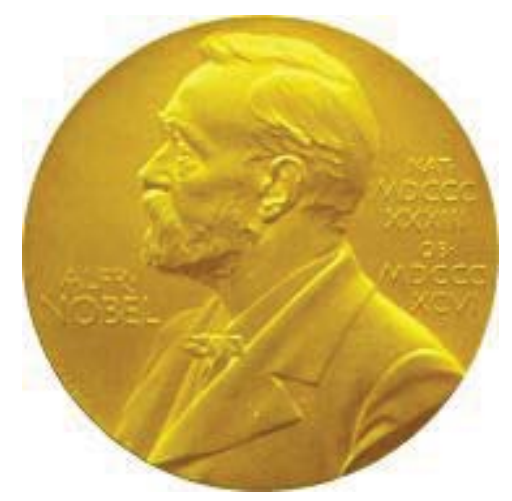

\section{COST EFFECTIVE - LOW RISK - MATURE TECHNOLOGIES}

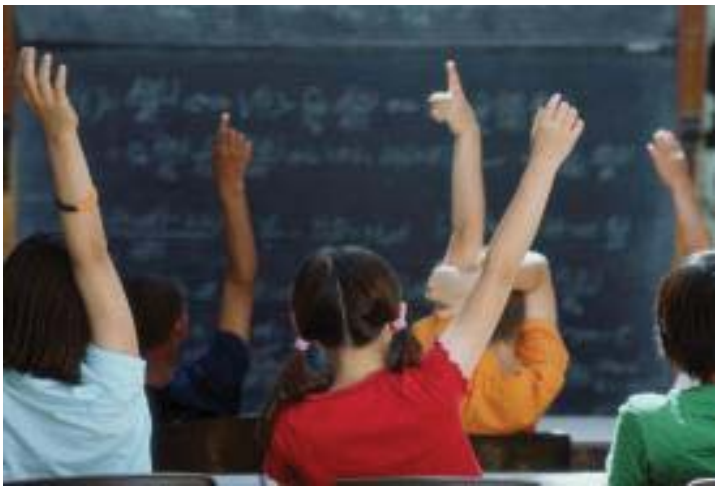

Brings Universe to STEM Next generation citizen science

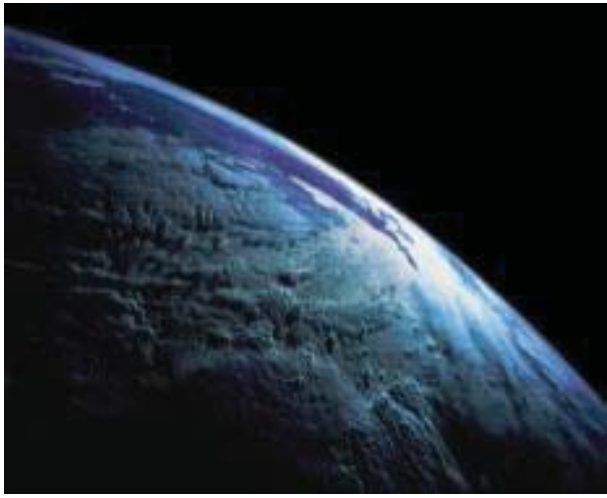

Foundation for discovering Earth-like planets

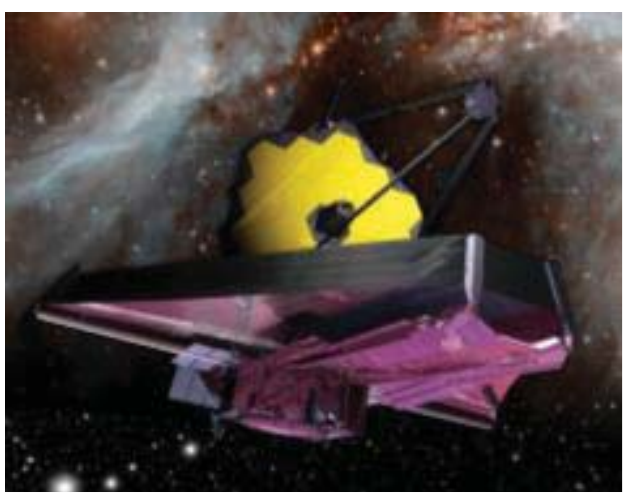

Complements and enhances JWST science 


\section{WFIST-AFTA Optical Design}

- Wide-Field Instrument composed of two channels:

- Wide-Field Channel (WFC) provides 1/3-square degree of instantaneous field coverage at 0.11 arcsecond pixel scale.

- WFC Focal plane of 300 million pixels

- Diffraction-limited imaging mode (WFI), operating in six panchromatic bands between $0.6-2.0 \mu \mathrm{m}$, or spectrographic mode (WFS) from 1.3-2.0 $\mu \mathrm{m}$.

- Separate Integral Field Channel (IFC) - Discrete spectral analysis over a 3"x3" field with $0.15^{\prime \prime}$ sampling and $R=100$ spectral resolution. 


\section{Wide-Field Channel Optical Design}

- The WFIRST-AFTA TMA optical design is anchored to the repurposed assets' size and figures

- Allows for small changes in the conic figure of the Primary Mirror (T1) and minor changes in the curvature and position of the Secondary Mirror (T2)

- Entrance Aperture Plate (EAP) at the intermediate focus passes the WFC's field into the instrument enclosure. 


\section{Wide-Field Channel Optical Design}

- Powered mirror within the instrument (M3) works in concert with T1 \& T2 to produce a corrected field

- All three powered mirrors are optically co-axial and simple conics

- Two fold mirrors are use for packaging

- Maximum root mean square (RMS) polychromatic wavefront error of $45 \mathrm{~nm}$ across entire field

- Half of the 90nm budget (based on 1/13 $\lambda @ 1.2 \mu \mathrm{m}$ )

- Currently in "Cycle 4" design iteration 


\section{Focal Plane Array Layout}

- 18 individual H4RG-10 detectors, each with 4,088x4,088 10 $\mathrm{mm}$ active pixels.

- Each sensor is custom shimmed in the array with a tilt and piston to match a field curvature of about 16 meters, allowing a 10\% performance gain over a perfectly flat field.

- 6 columns of 3 sensors w/ offset columns

- Columns follow the natural annular curve of an off-axis TMA optimized field. 


\section{Pupil / Element Wheel}

- At the pupil, located approximately midway between M3 and the FPA, is the cold/Lyot stop and the element wheel.

- Blocks the scatter and emissions form the telescope struts and baffles.

- 8-position Element Wheel: 6 bandpass filters, 1 spectrograph grism assembly, 1 null position.

- All wheel modes are par-focal to no filter.

- The filters are very weak meniscus lenses.

- The grism consists of 3 optical elements; only spherical surfaces on fused silica and two diffracting surfaces on flats. (See presentation by Qian Gong) 


\section{Optical Design Considerations}

- Constraints of existing hardware (Use "as-is")

- Hold volume while maintaining large field.

- Pupil clearance to allow for the large element wheel

- M3 distance to allow for mounting

- EAP location which allows for clean baffling of the intermediate focus

- Minimum central obscuration 


\section{W】IDR - TR Wide Field Infrared Survey Telescope - AFTA}

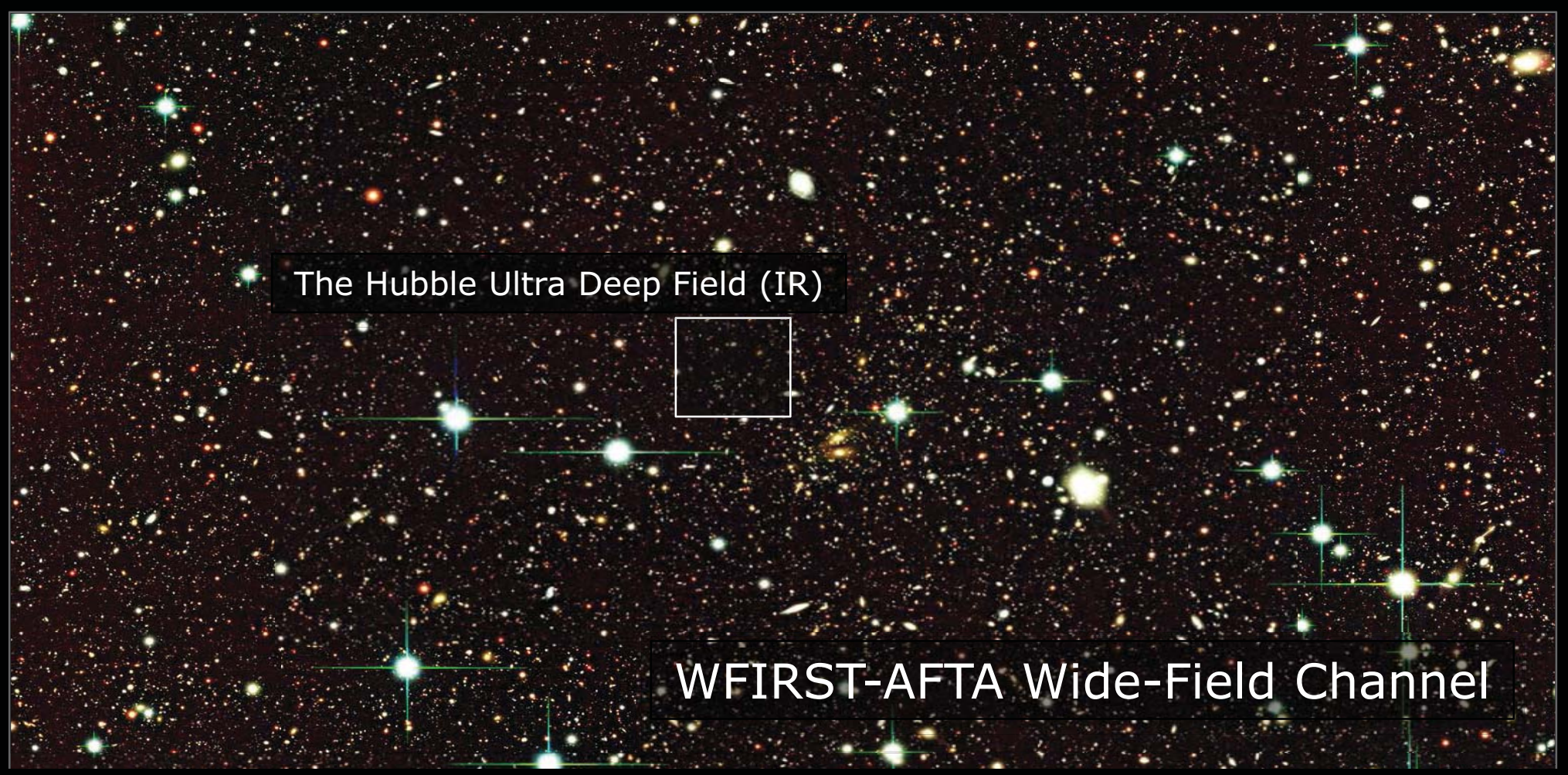

Imagine $200 x$ more, with $>1,000,000$ galaxies (a $20^{\prime} \times 10^{\prime}$ wall of "retina" displays!)

Imagine this wall of a million galaxies - a 300 megapixel image from a single WFIRST-AFTA pointing -- filling walls of schools and museums and providing a wealth of citizen science. 


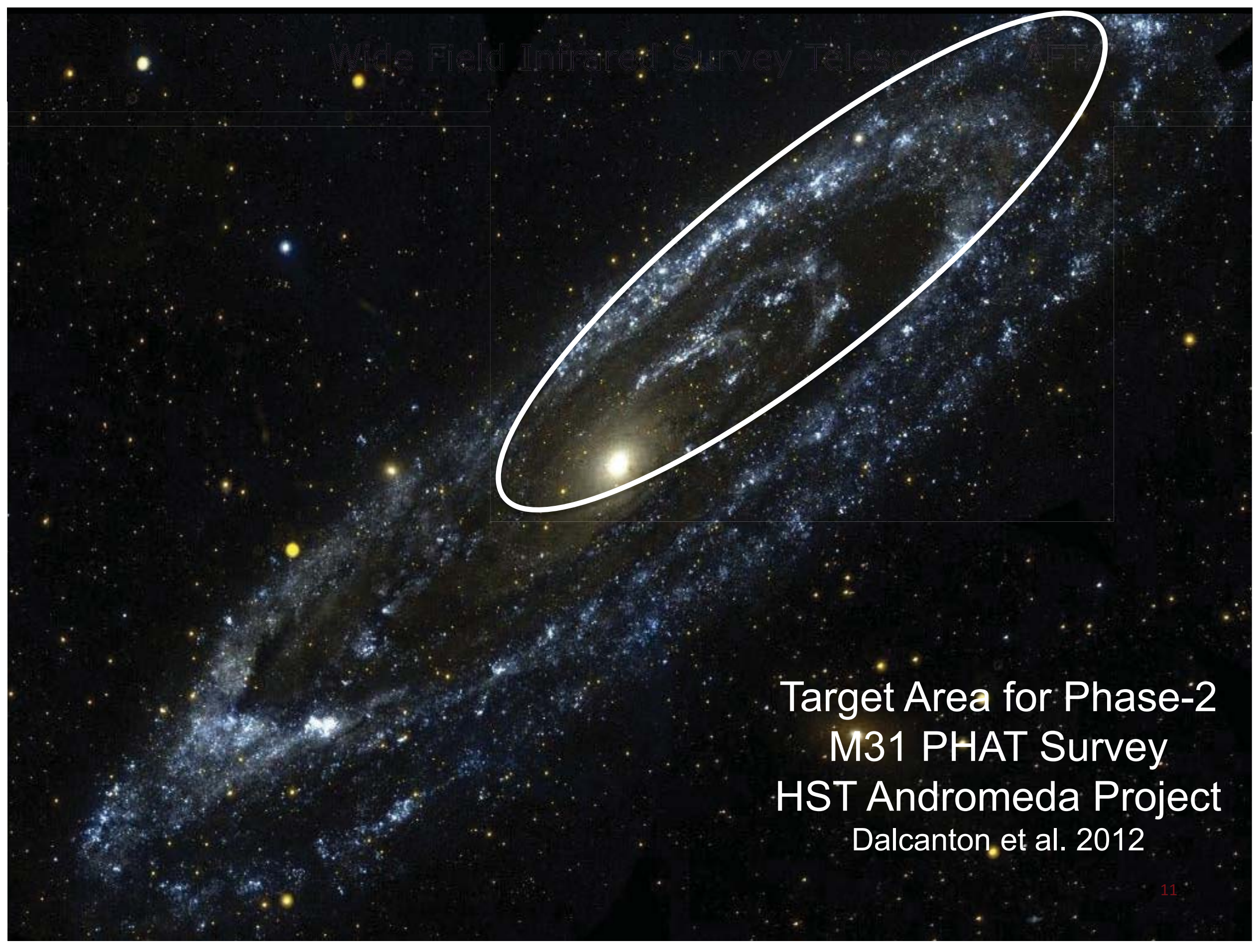




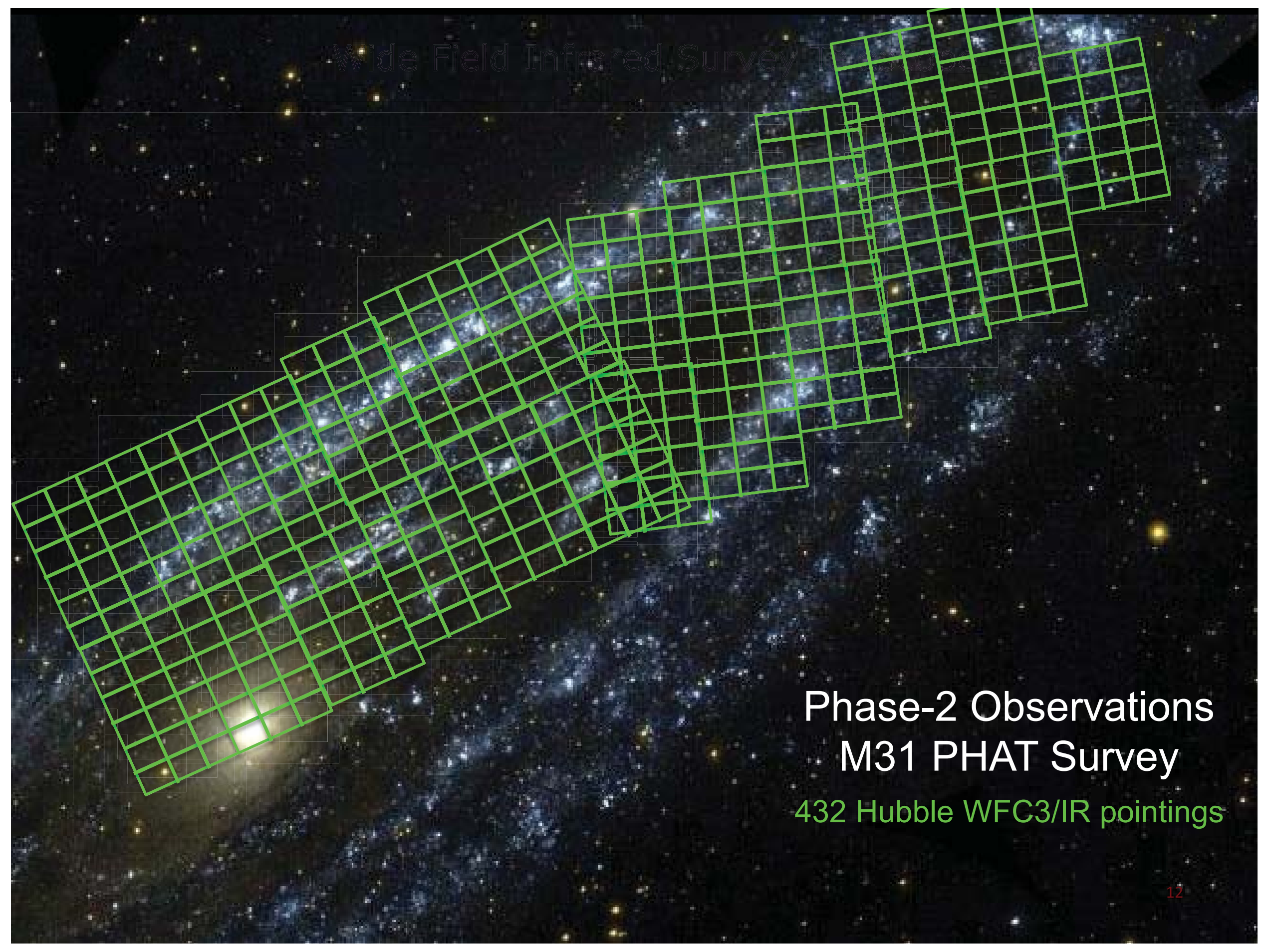




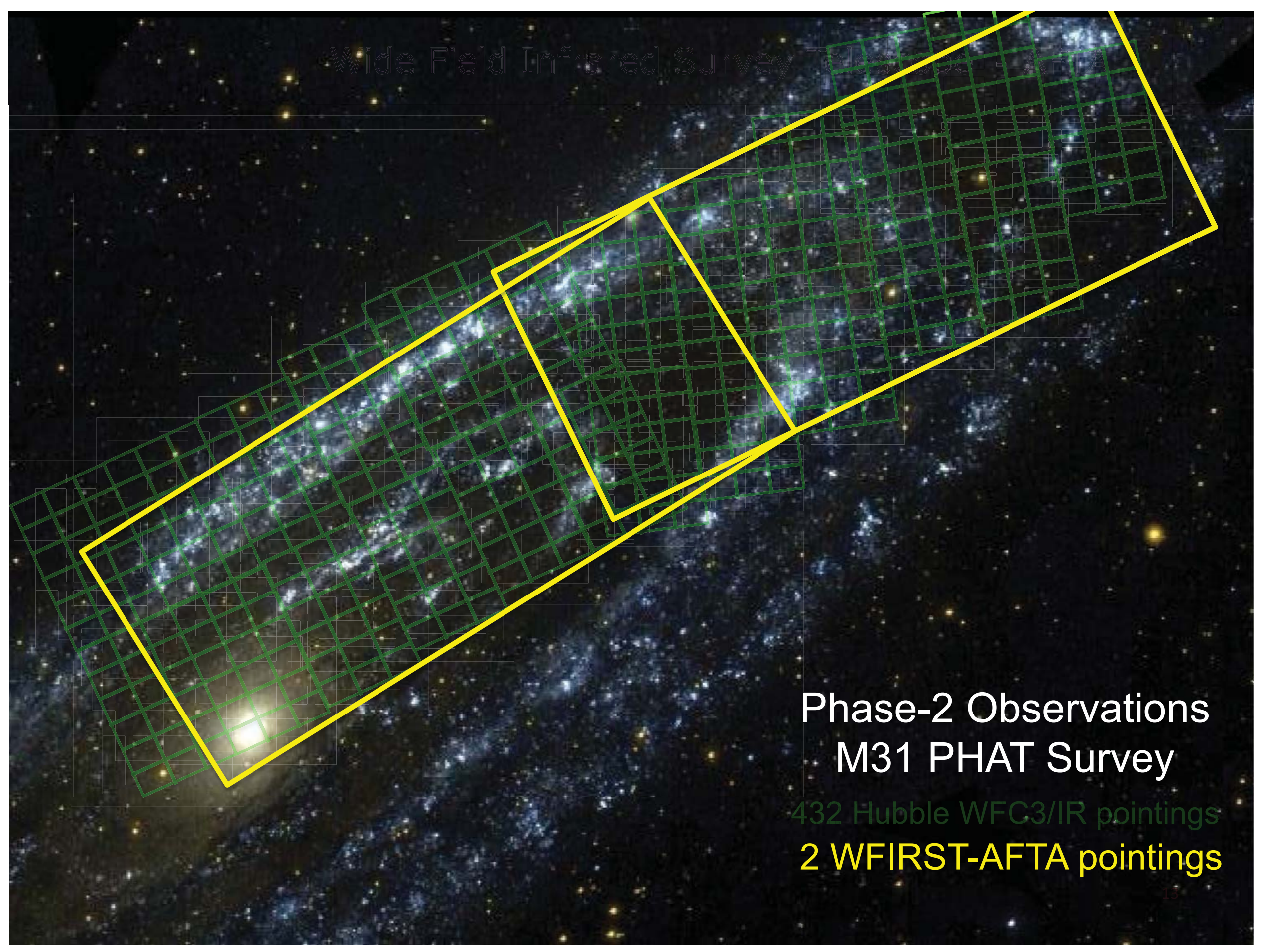




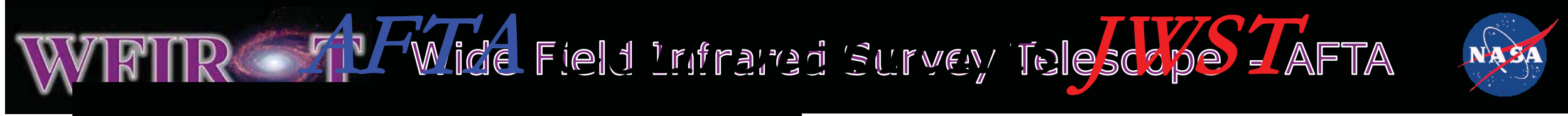
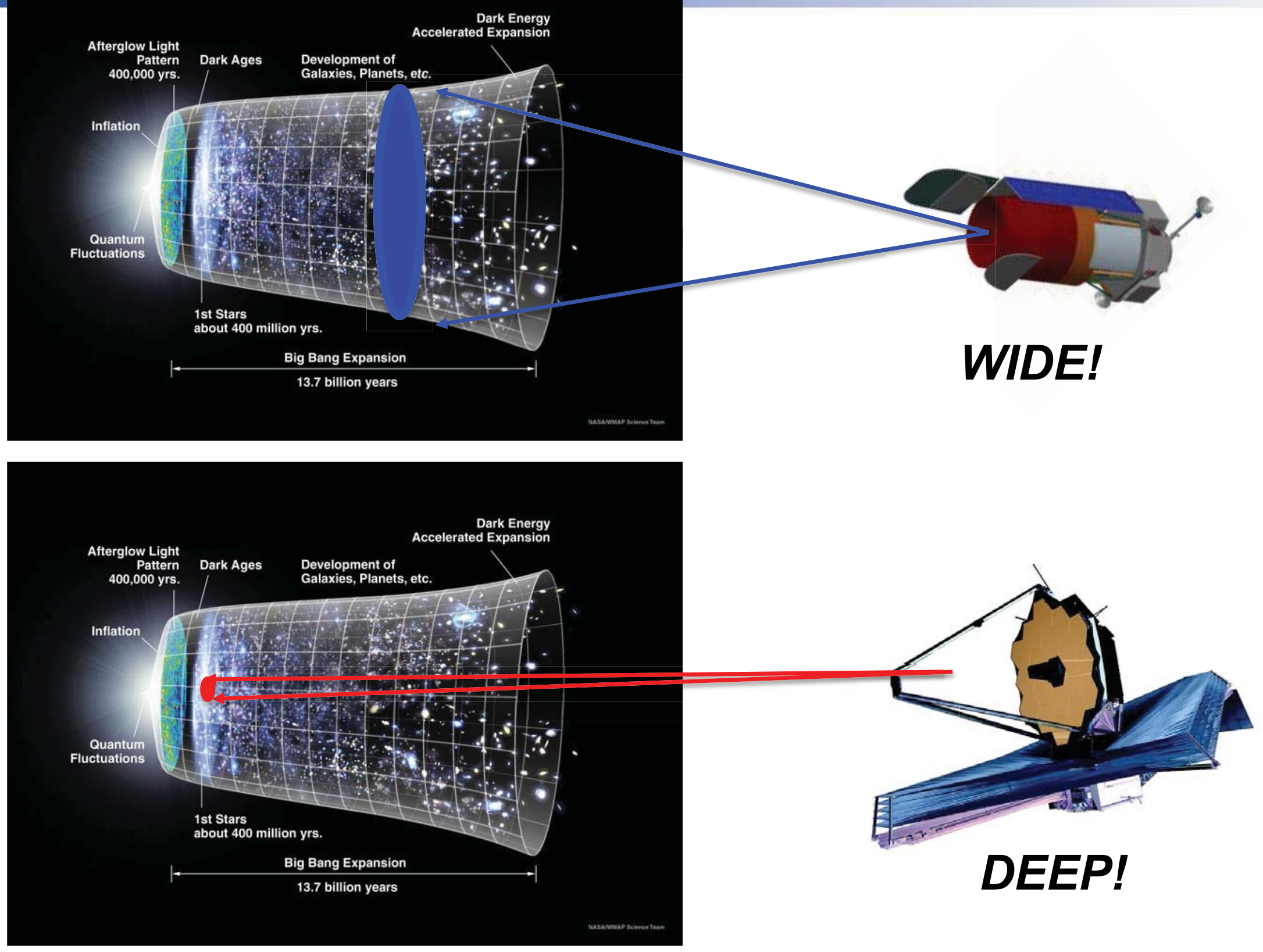

WIDE! 


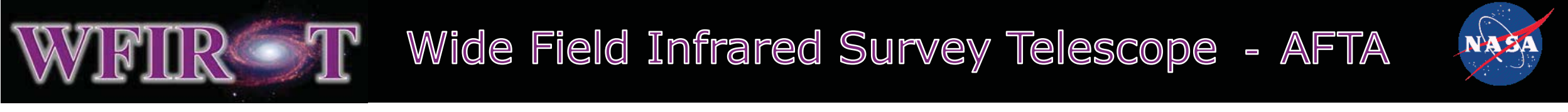

\section{WFIRST Observatory Layout}

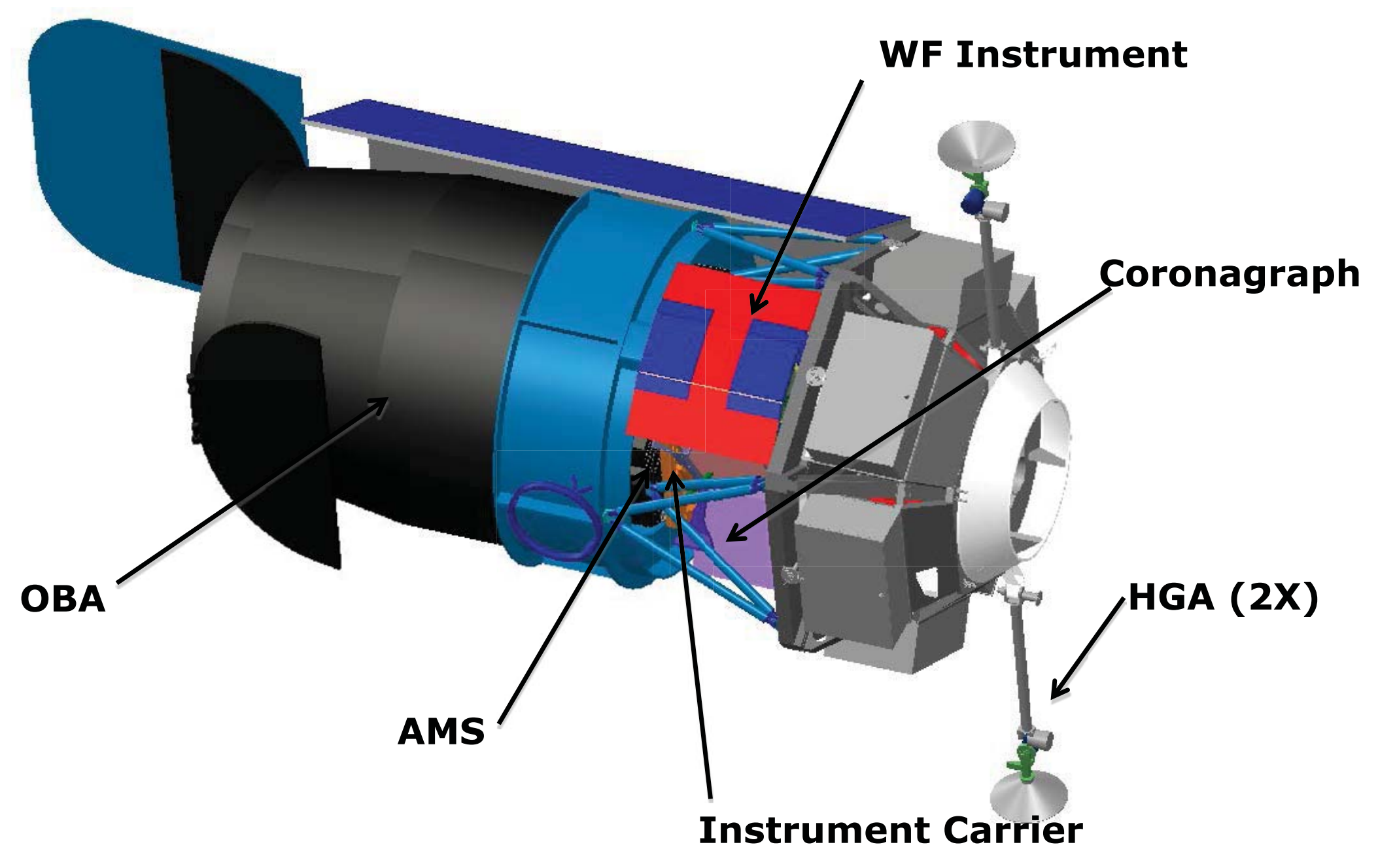




\section{Wide Field Infrared Survey Telescope = AFTA}

\section{Telescope Reuse}

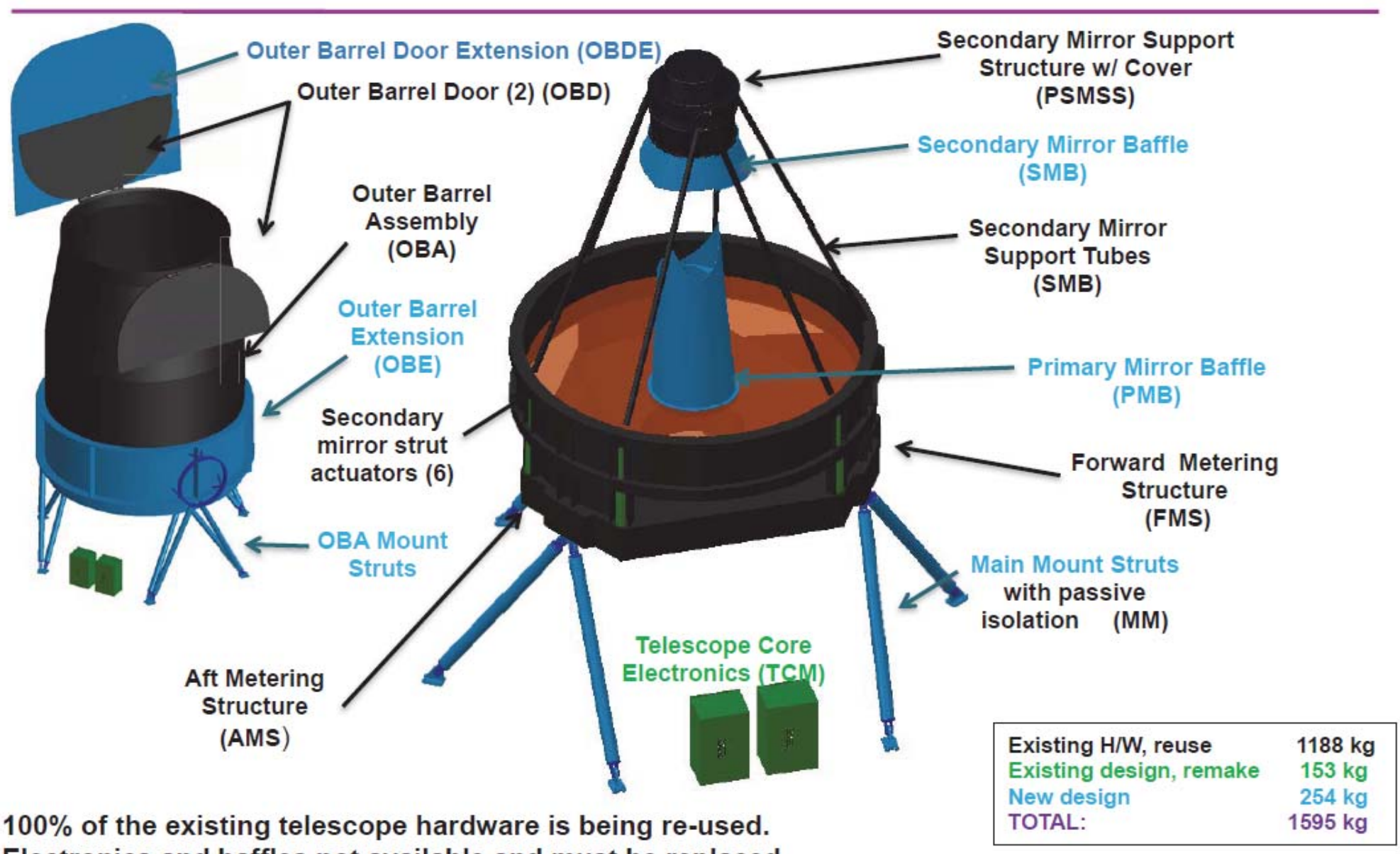

Electronics and baffles not available and must be replaced. 


\section{WIIMR - IT Wide Field Infrared Survey Telescope - AFTA Nigh}

\section{WFIRST IC/WFI/CGI Layout - Cycle 4}

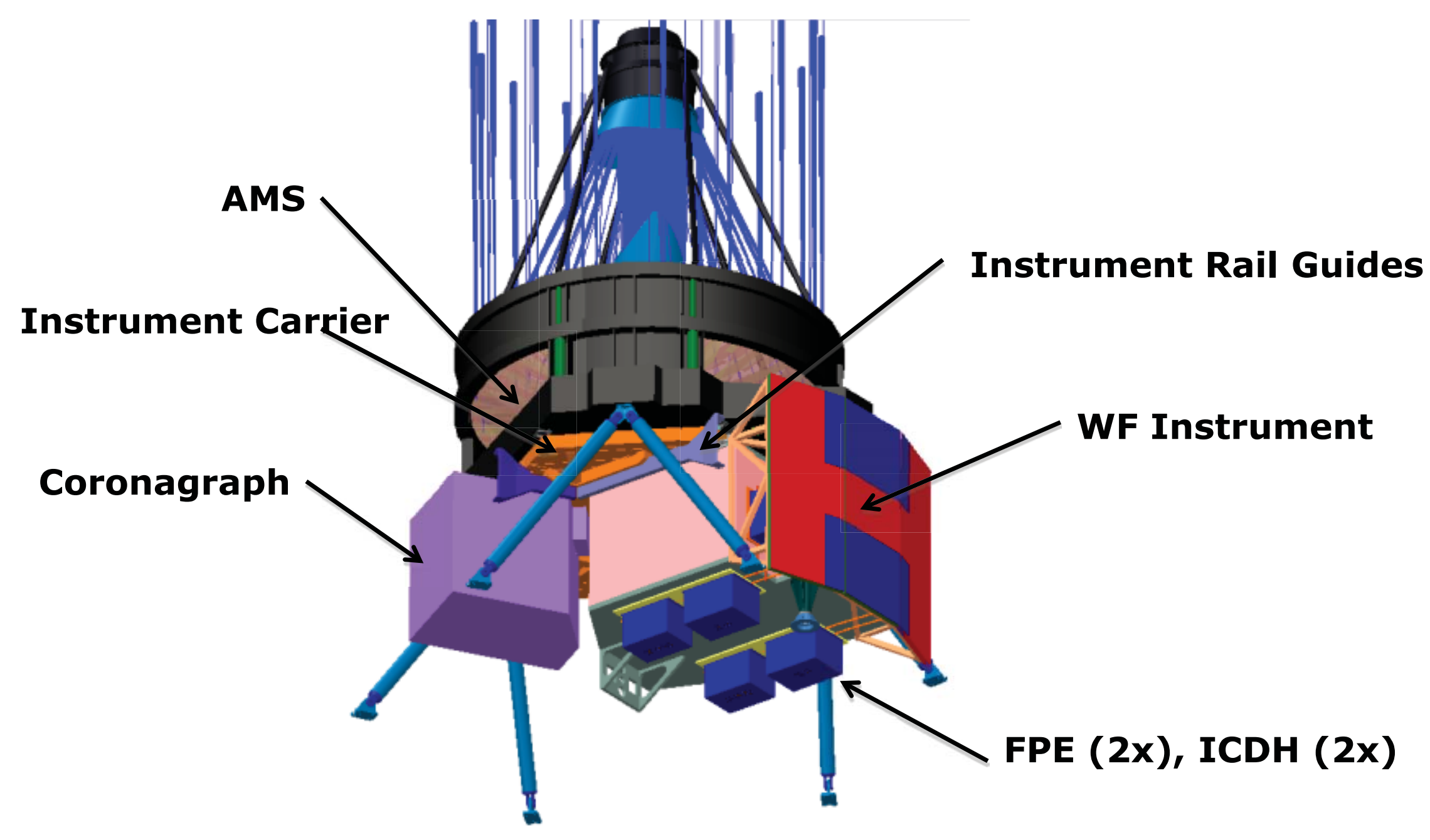




\section{Wide Field Infrared Survey Telescope - AFTA}

- Single wide field channel instrument

- 3 mirrors, 1 powered

- $184 \mathrm{~K}$ x 4K HgCdTe detectors

- 0.11 arc-sec plate scale

- IFU for SNe spectra, single $\mathrm{HgCdTe}$ detector

- Single filter wheel

- Grism used for GRS survey

- Thermal control passive radiator

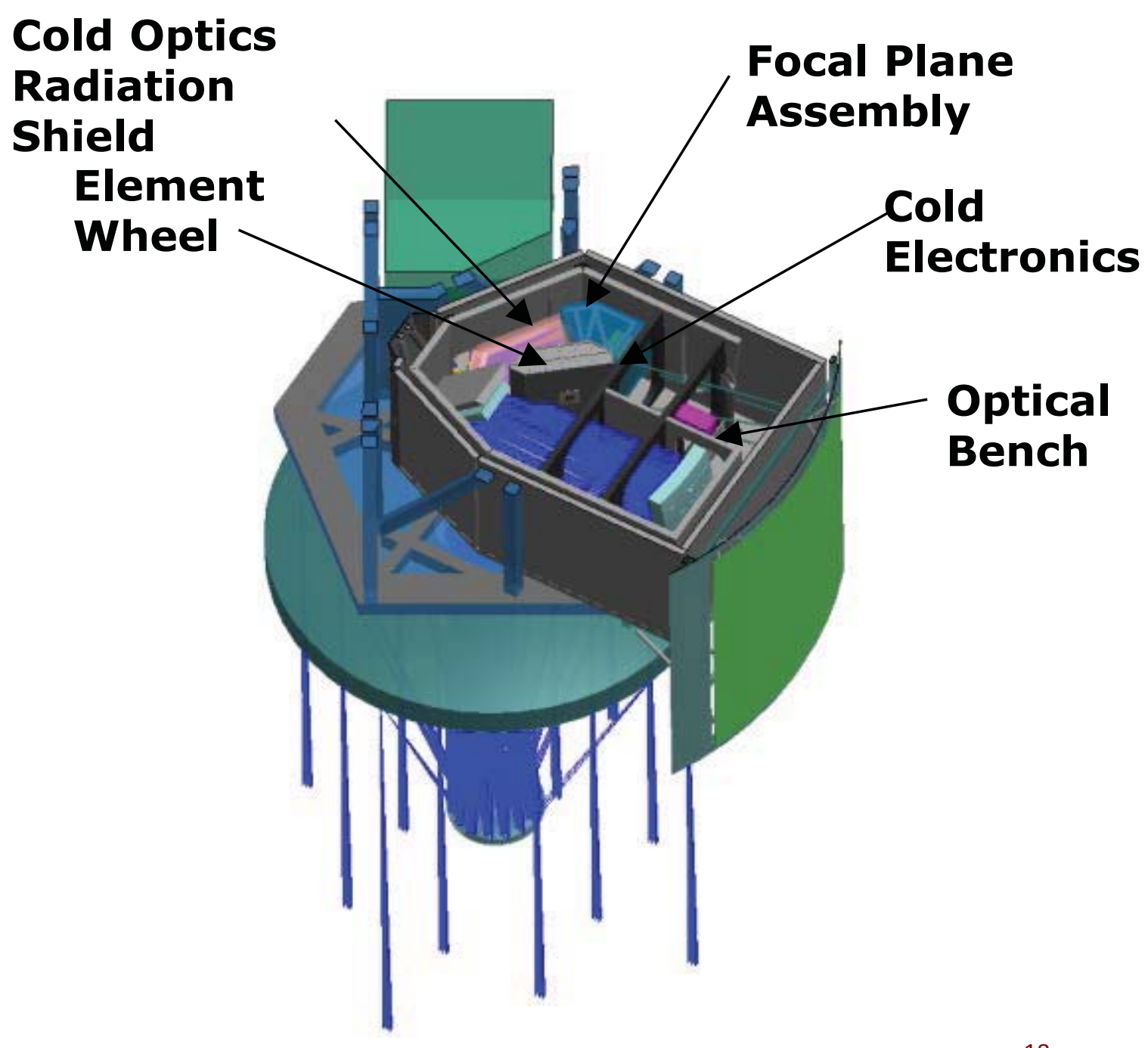

Cold Optics Radiation Element Wheel 


\section{WWITIR $\subset$ TI Wide Field Infrared Survey Telescope - AFTA}

\section{WFIRST WFI Layout - Cycle 4}

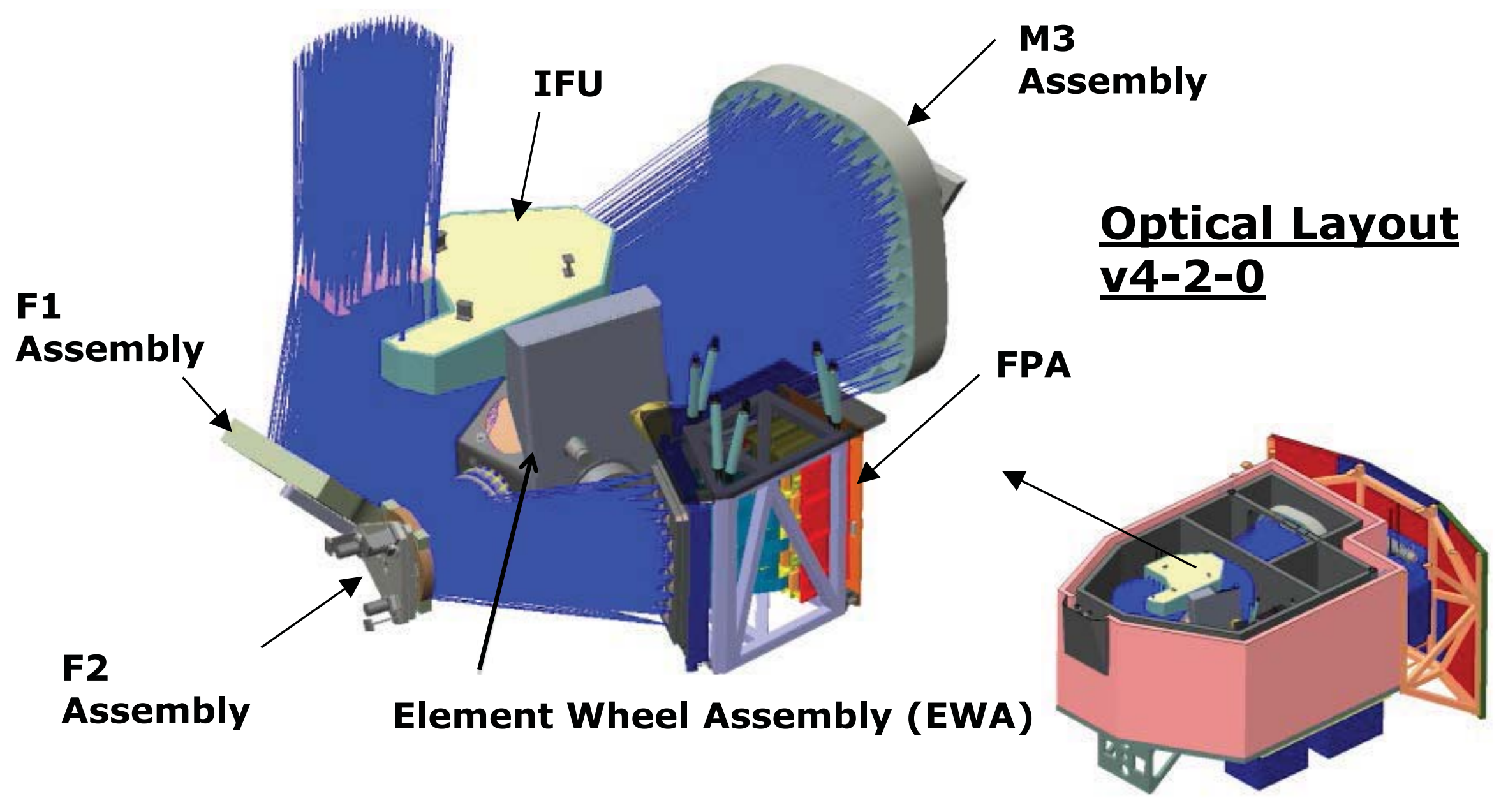




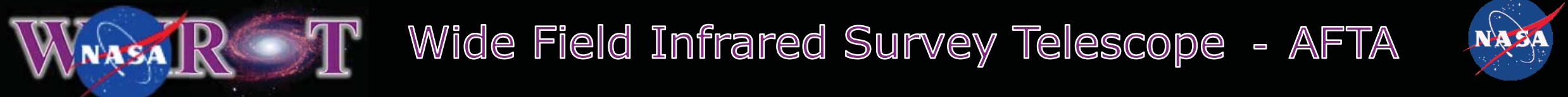

\section{Wide field Instrument Shares Architecture and Heritage with HST/WFC3}
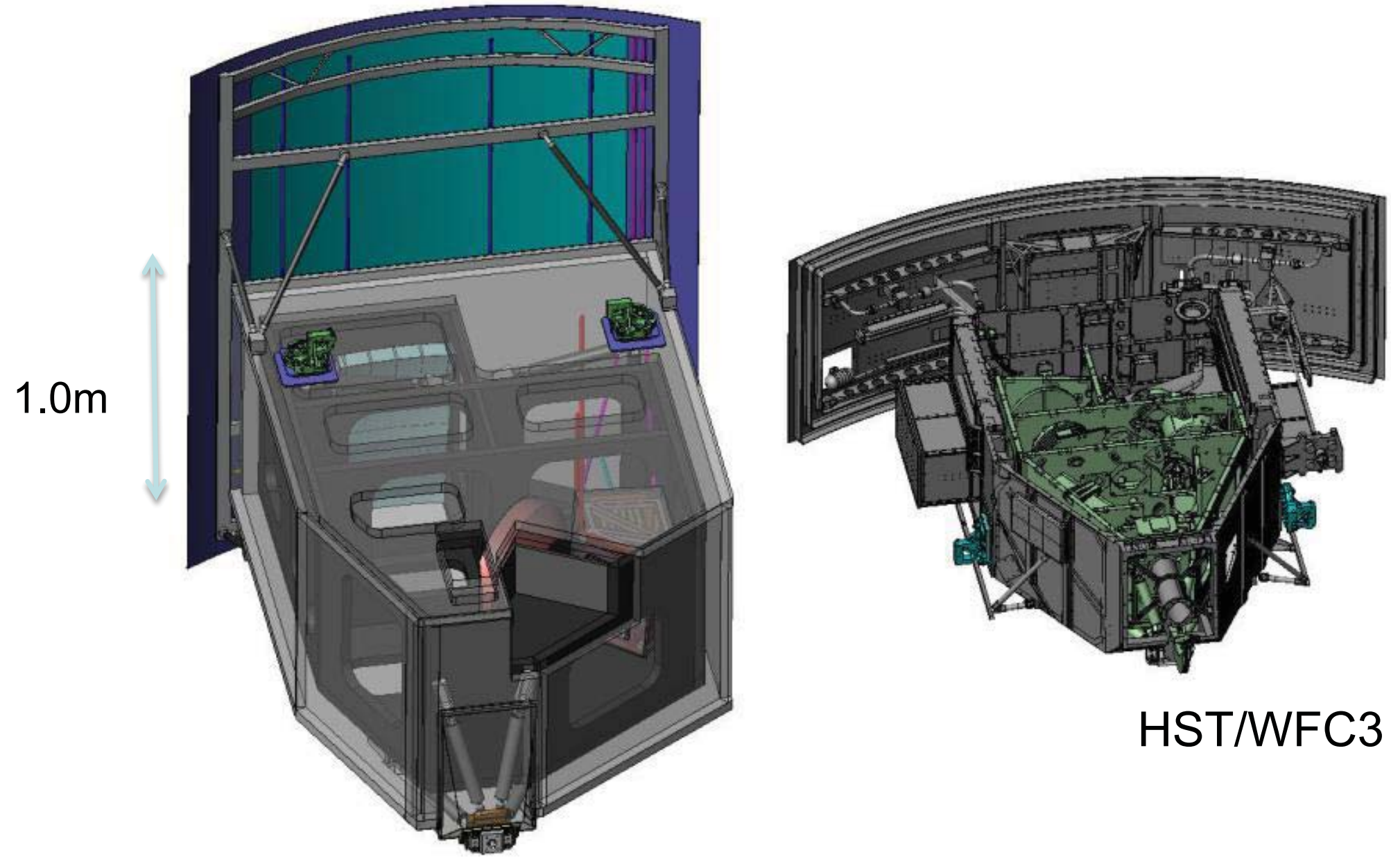

HST/WFC3

WFIRST wide field 


\section{WVIFIR GT Wide Field Infrared Survey Telescope - AFTA}

\section{Wide-Field Major Subassemblies}

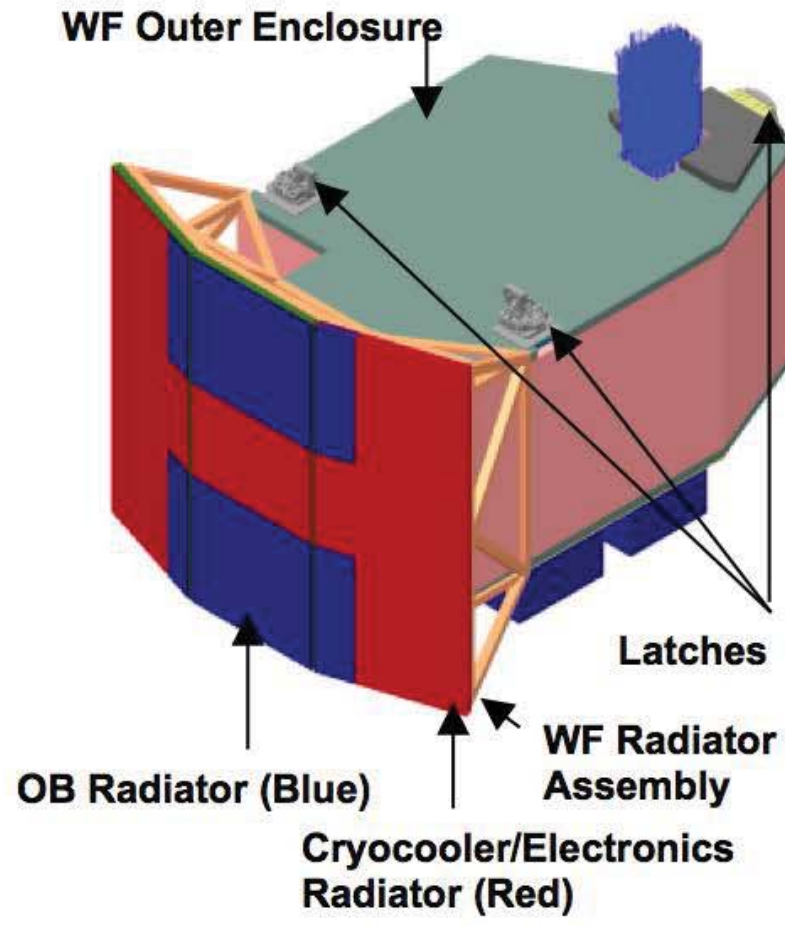

Radiator (Red)

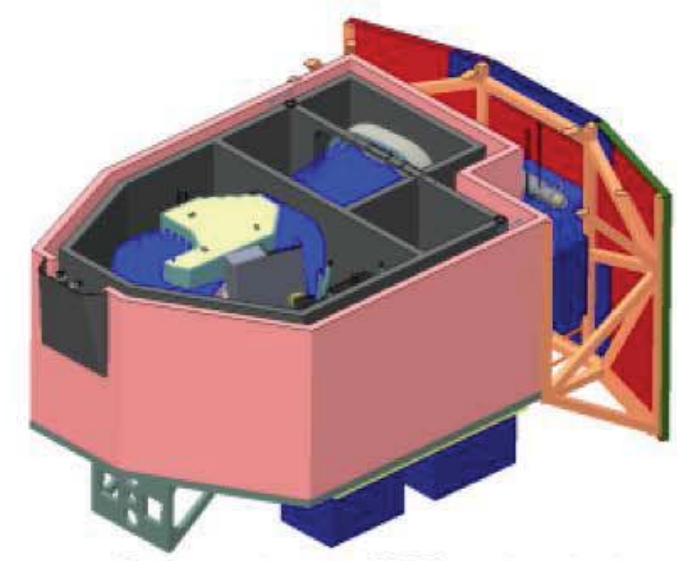

Outer enclosure (OE) and optical bench (OB) top panels removed

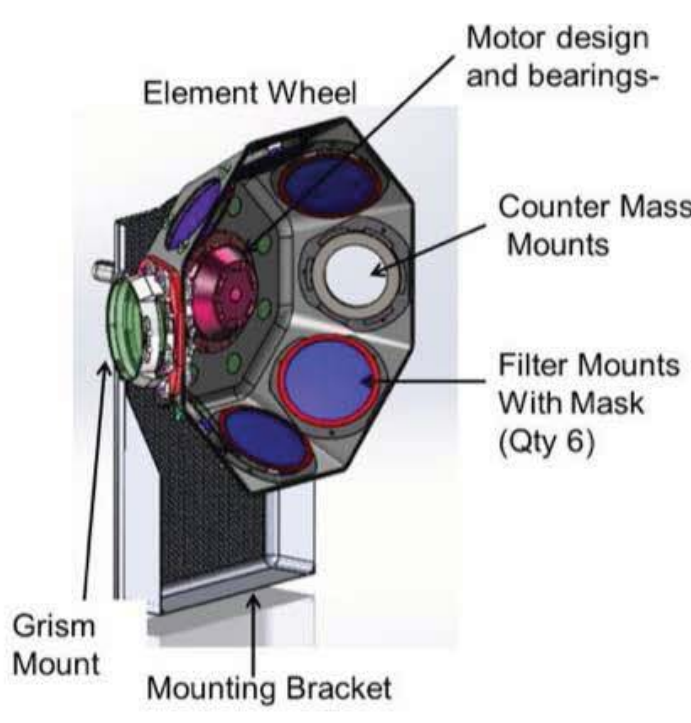

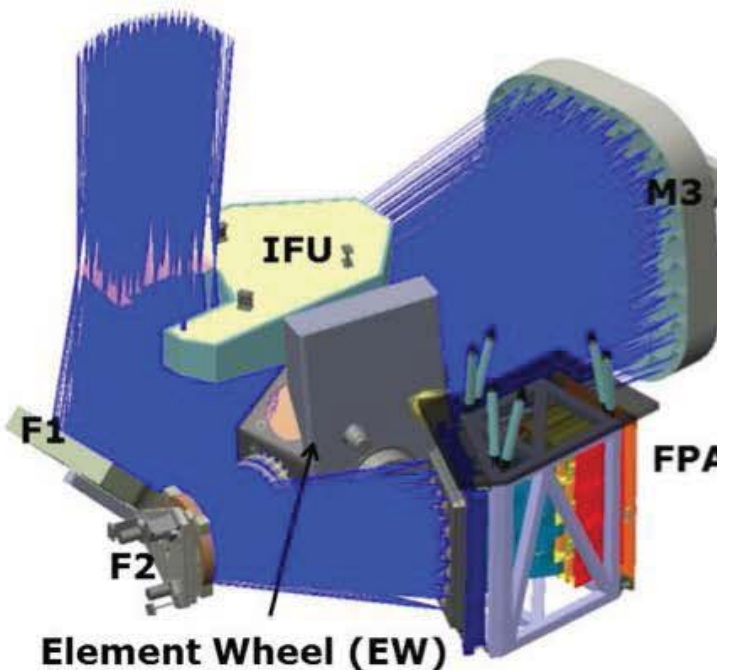

Wide field mirrors; Focal plane assembly (FPA); Integral field unit (IFU)

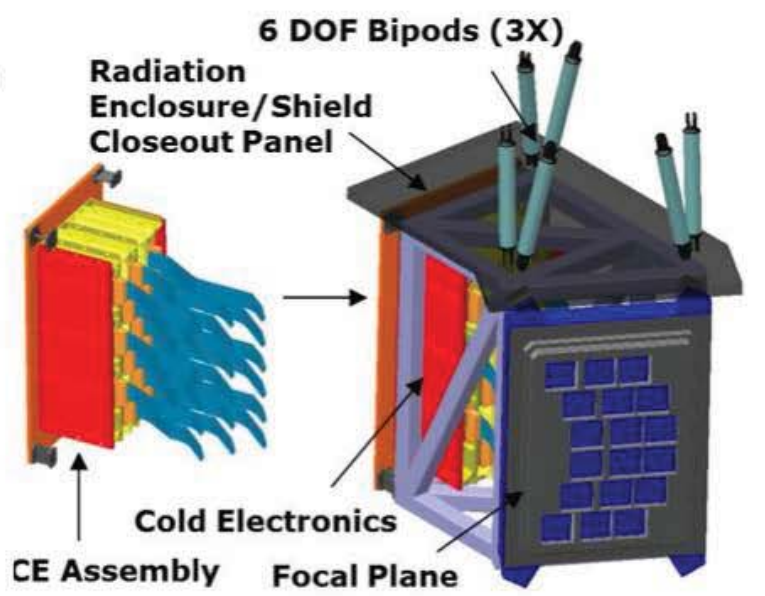




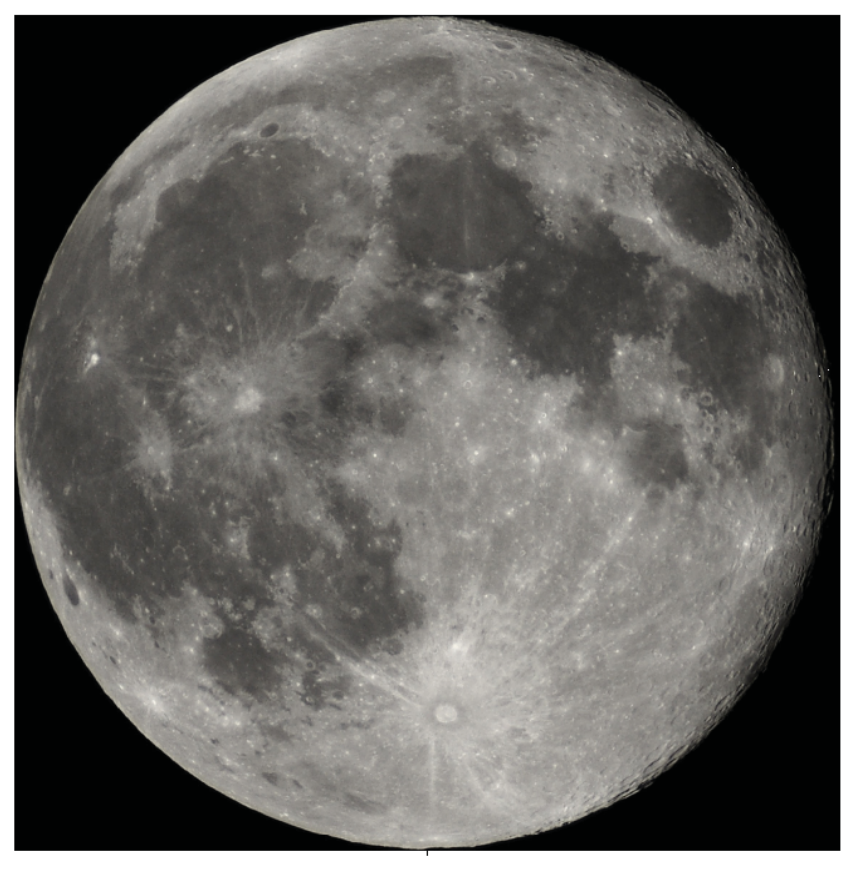

Moon (average size seen from Earth)
18 NIR detectors
$0.11 \mathrm{arcsec} / \mathrm{pixel} \quad 0.28 \mathrm{deg}^{2}$
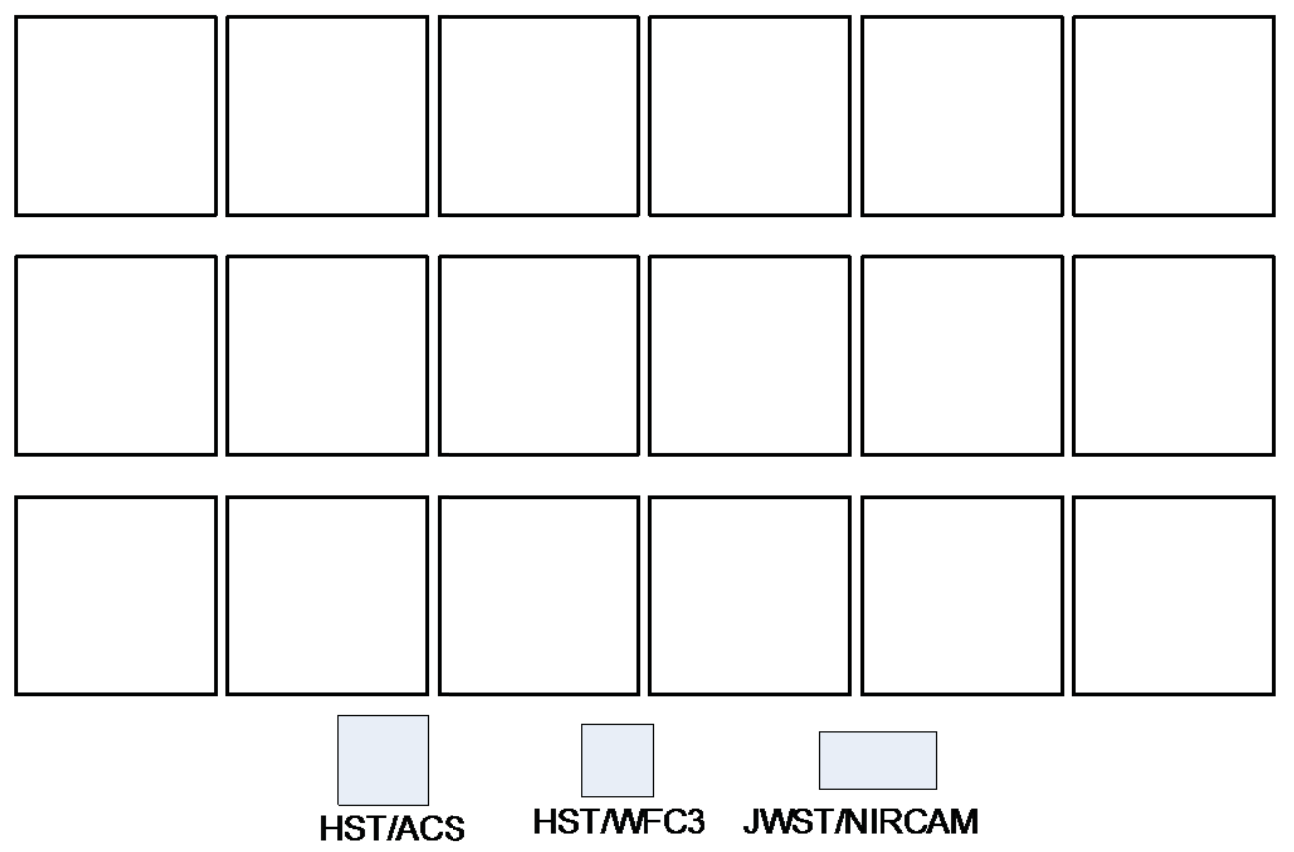

Each square is a H4RG-10 $4 \mathrm{k} \times 4 \mathrm{k}, 10$ micron pitch 288 Mpixels total

Slitless spectroscopy with grism in filter wheel R_ $\theta \sim 100$ arcsec/micron 


\title{
OPTICAL DESIGN OF WFIRST-AFTA WIDE-FIELD INSTRUMENT
}

\author{
Bert Pasquale \\ NASA Goddard Space Flight Center \\ CONTINUED \\ PART 2
}




\section{W!}

\section{Design overview}

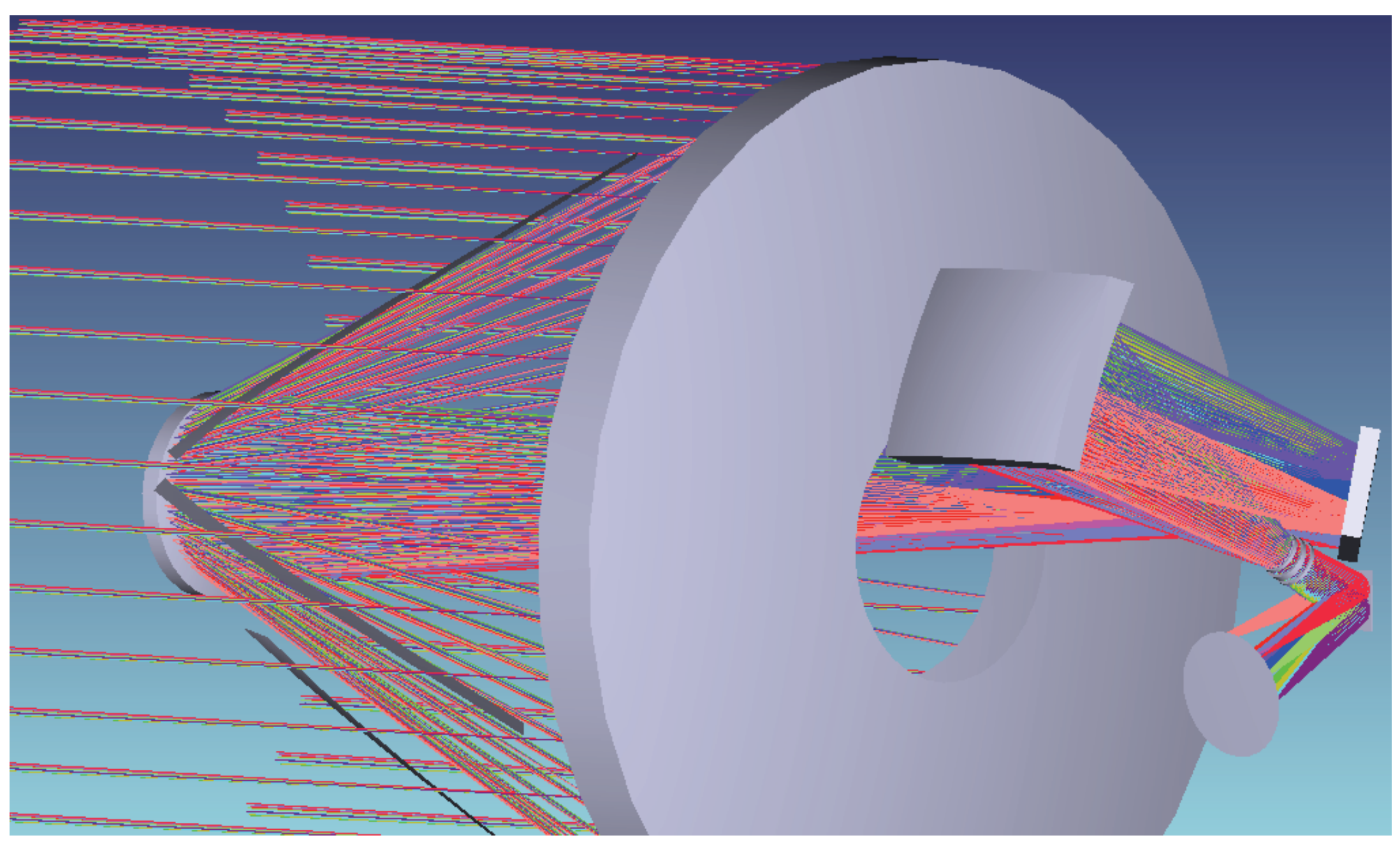


WIIR - T Wide Field Infrared Survey Telescope - AFTA NASA

\section{ATLAST (v4.2.5) Optical Components}

\begin{tabular}{|c|c|c|c|c|}
\hline & Radius (mm) & Conic & $\begin{array}{c}\text { Aperture }(\mathrm{mm}) \\
\text { Circular: Diameter } \\
\text { Rectangular: Width } \times \text { Height }\end{array}$ & Other \\
\hline PM (T1) & $5,670.69$ & -0.97198 & C 2,362 & Concave \\
\hline SM (T2) & $1,294.73$ & -1.69502 & C 536 & Convex \\
\hline Fold 1 & Flat & 0 & $R 504 \times 280$ & \\
\hline Mirror 3 & $1,729.732$ & -0.56660 & $\begin{array}{l}R 620 \times 500 \\
240 \text { Off-Axis }\end{array}$ & Concave \\
\hline Filter & $\begin{array}{l}\text { S1: } 1,594.07 \\
\text { S2: } 1,593.09\end{array}$ & 0 & C 110 & Meniscus \\
\hline Fold 2 & Flat & 0 & C180 & \\
\hline
\end{tabular}




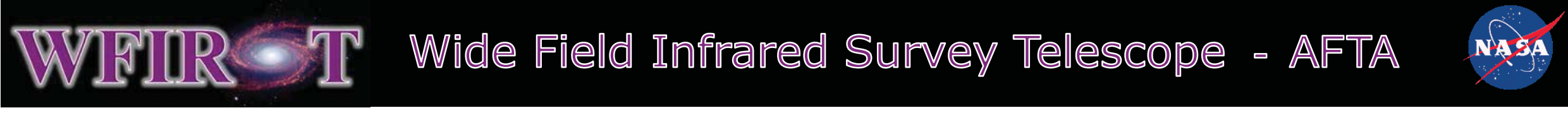

\section{Instrument ray trace views}
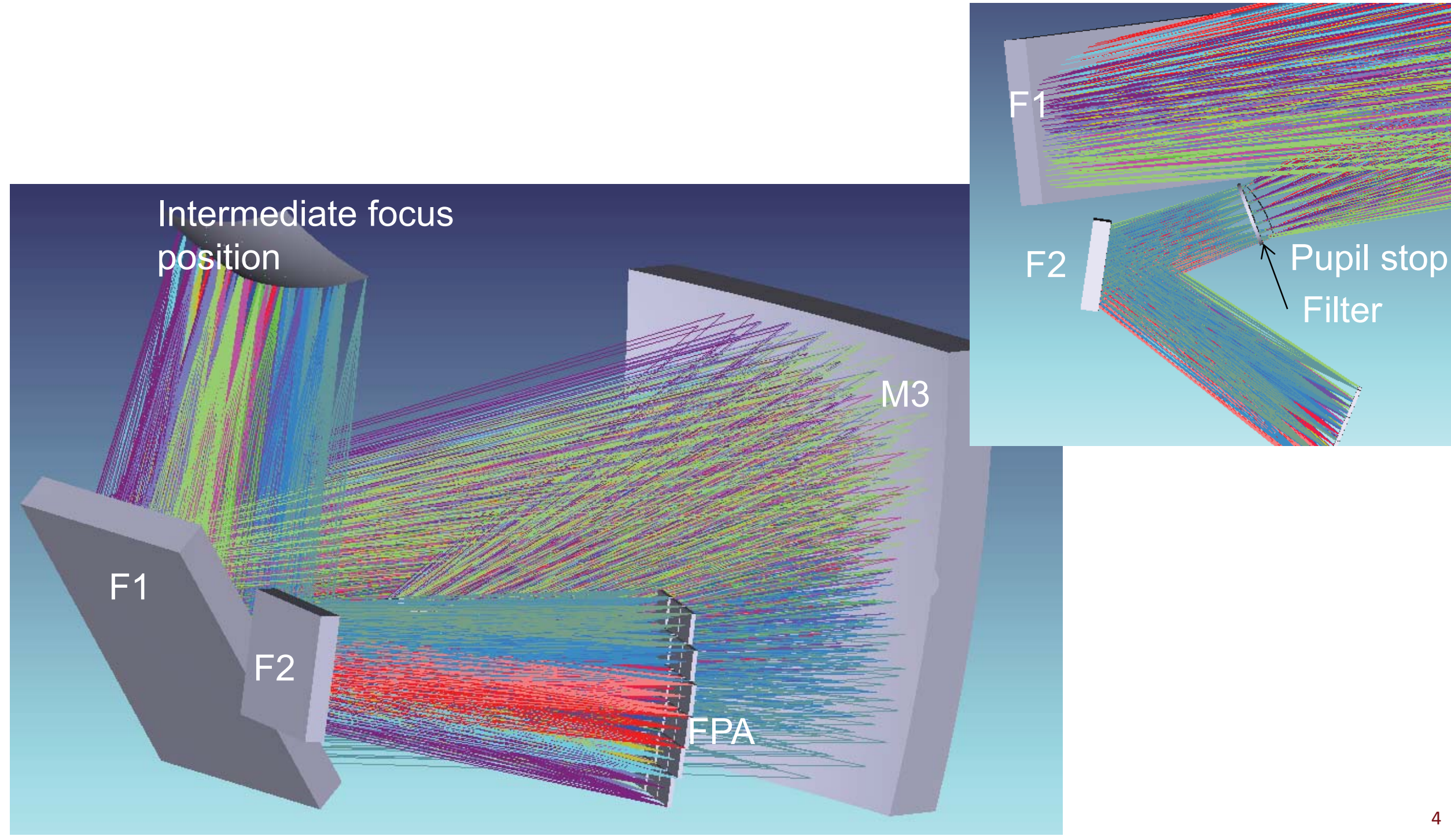


\section{WDITR - TR Wide Field Infrared Survey Telescope - AFTA NAga}

\section{Channel Field Layout for AFTA-WFIRST Instruments}

As Projected on Sky

[ Cycle 4, v4.2.5]

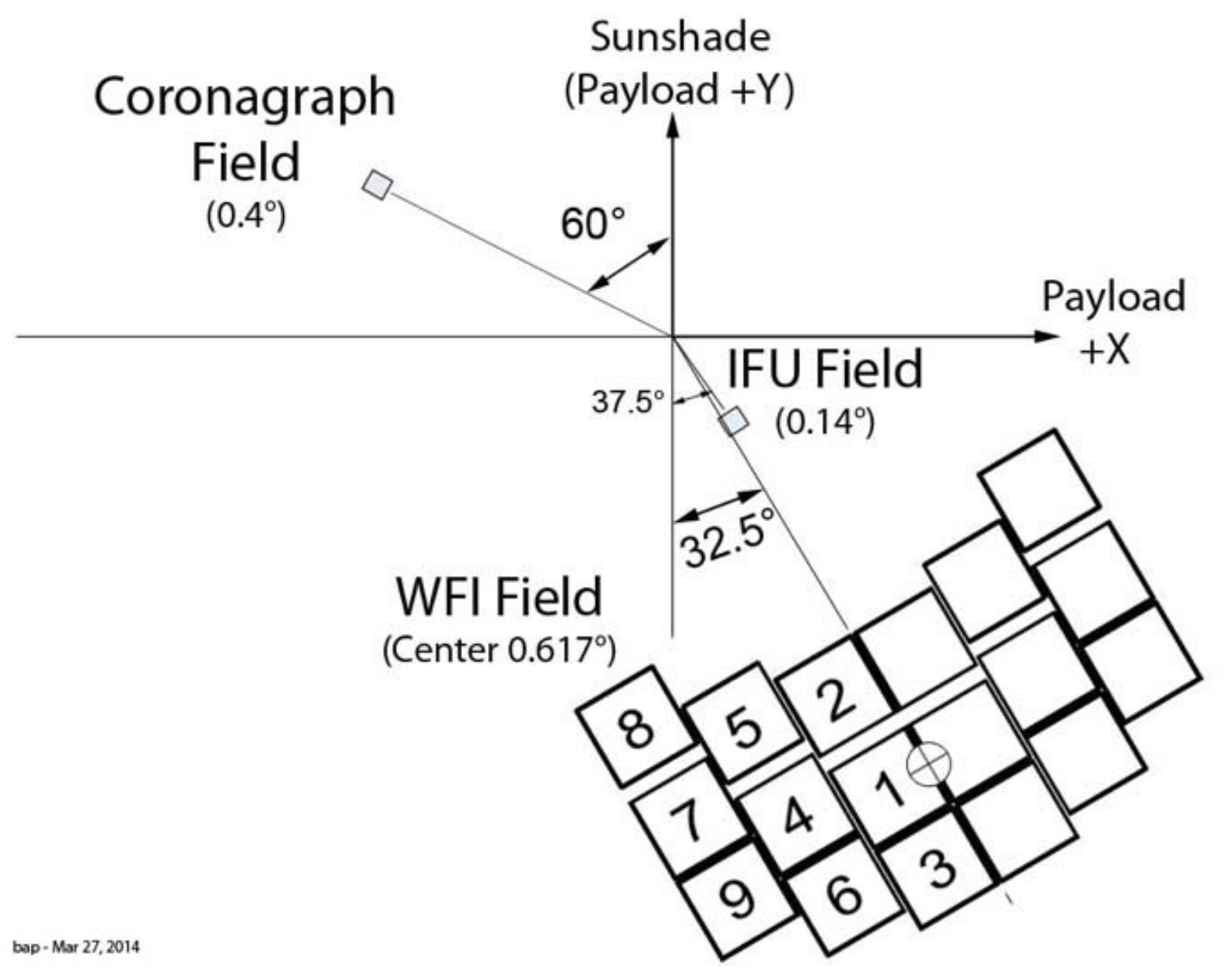




\section{Element Wheel Modes}

Wide-Field Imaging Mode Bandpass Filter

(6 positions)

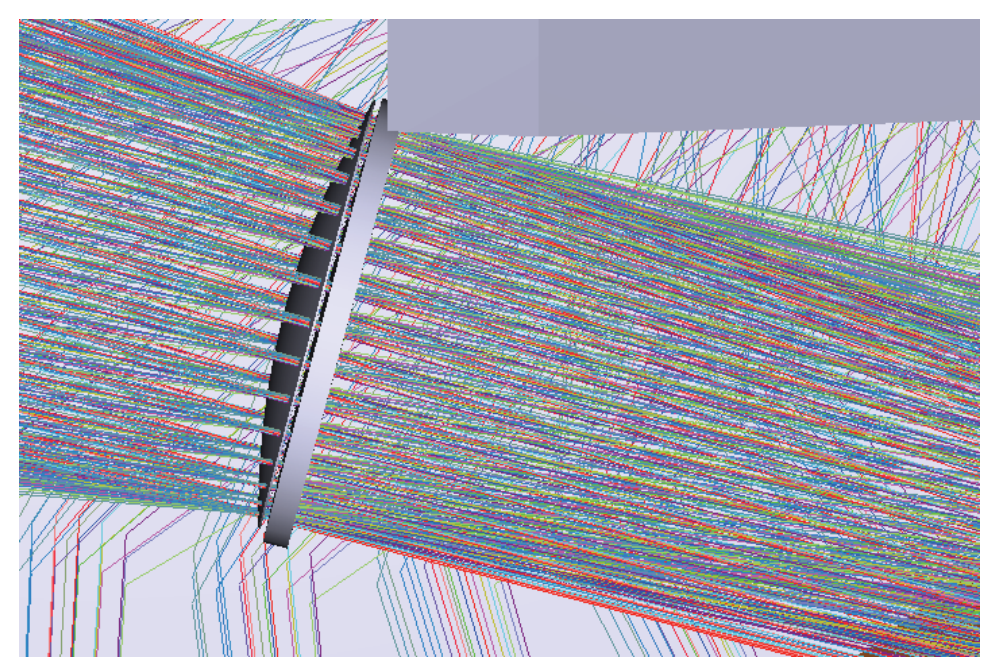

Wide-Field Spectrograph Mode Bandpass Filter

(1 position)

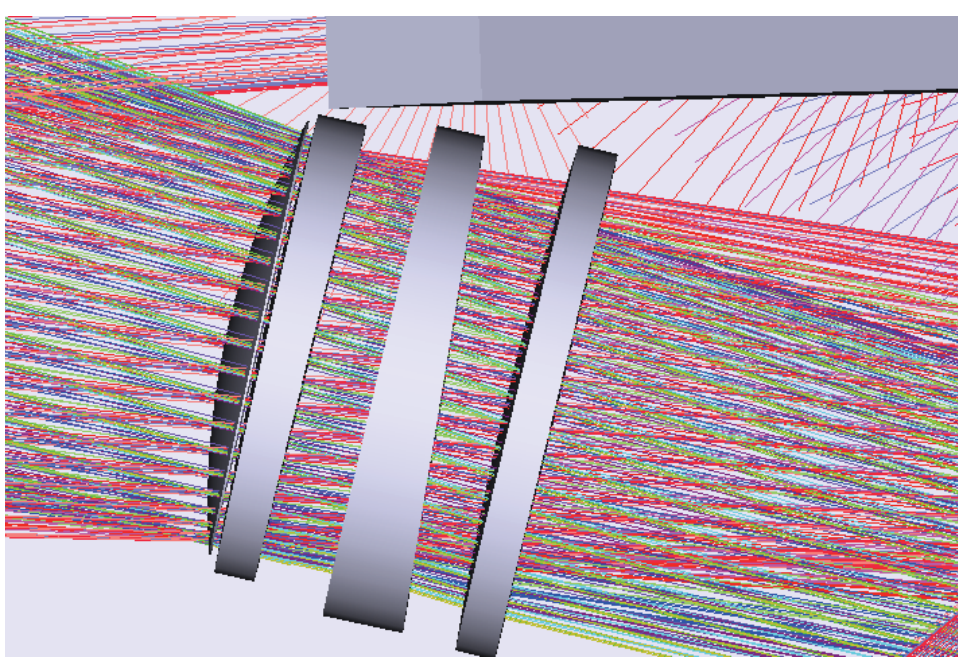




\section{WUISUR $\subseteq$ T Wide Field Infrared Survey Telescope - AFTA}

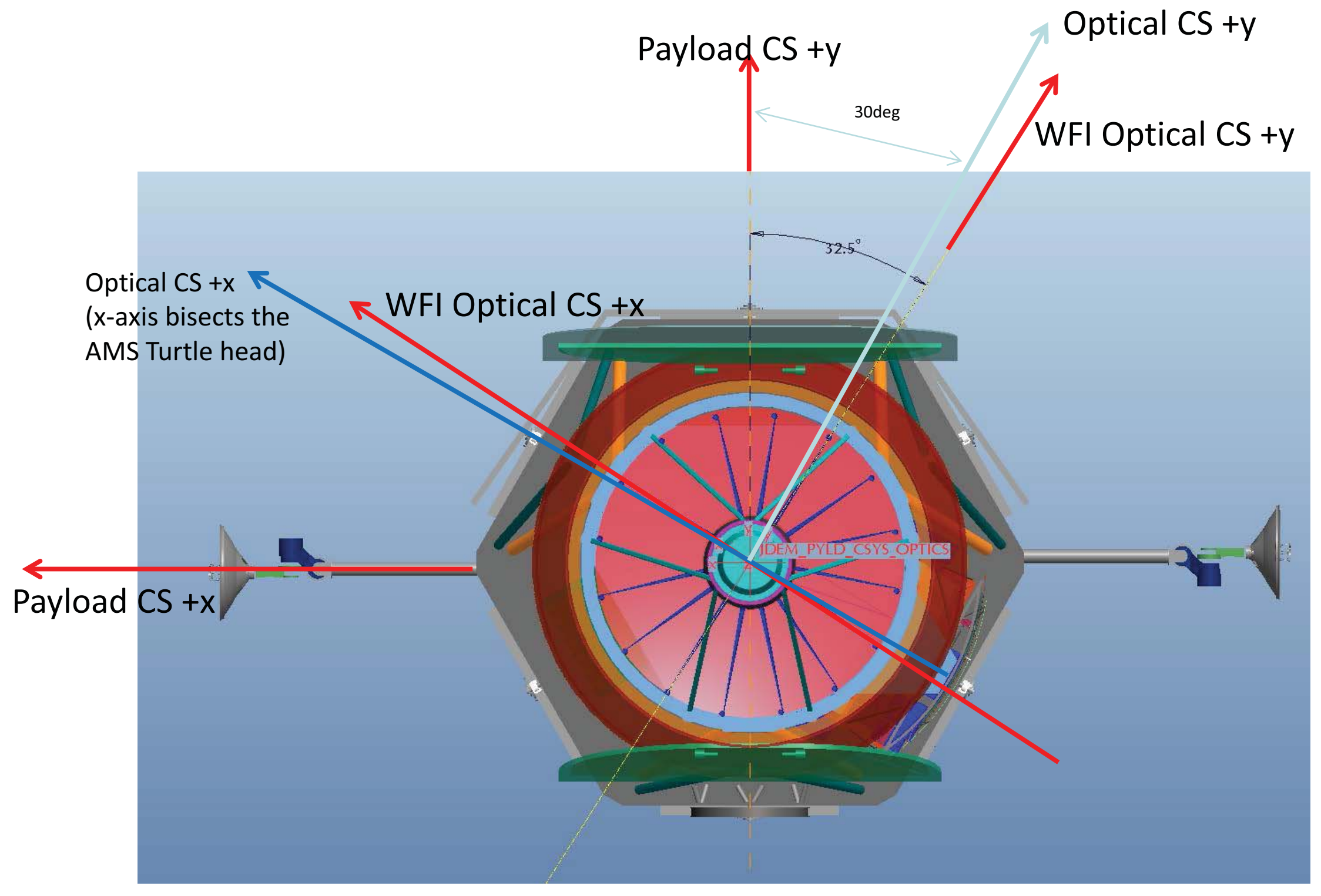




\section{WWIIR - TI Wide Field Infrared Survey Telescope - AFTA Ningy}

\section{Lyot Cold Stop Layout}

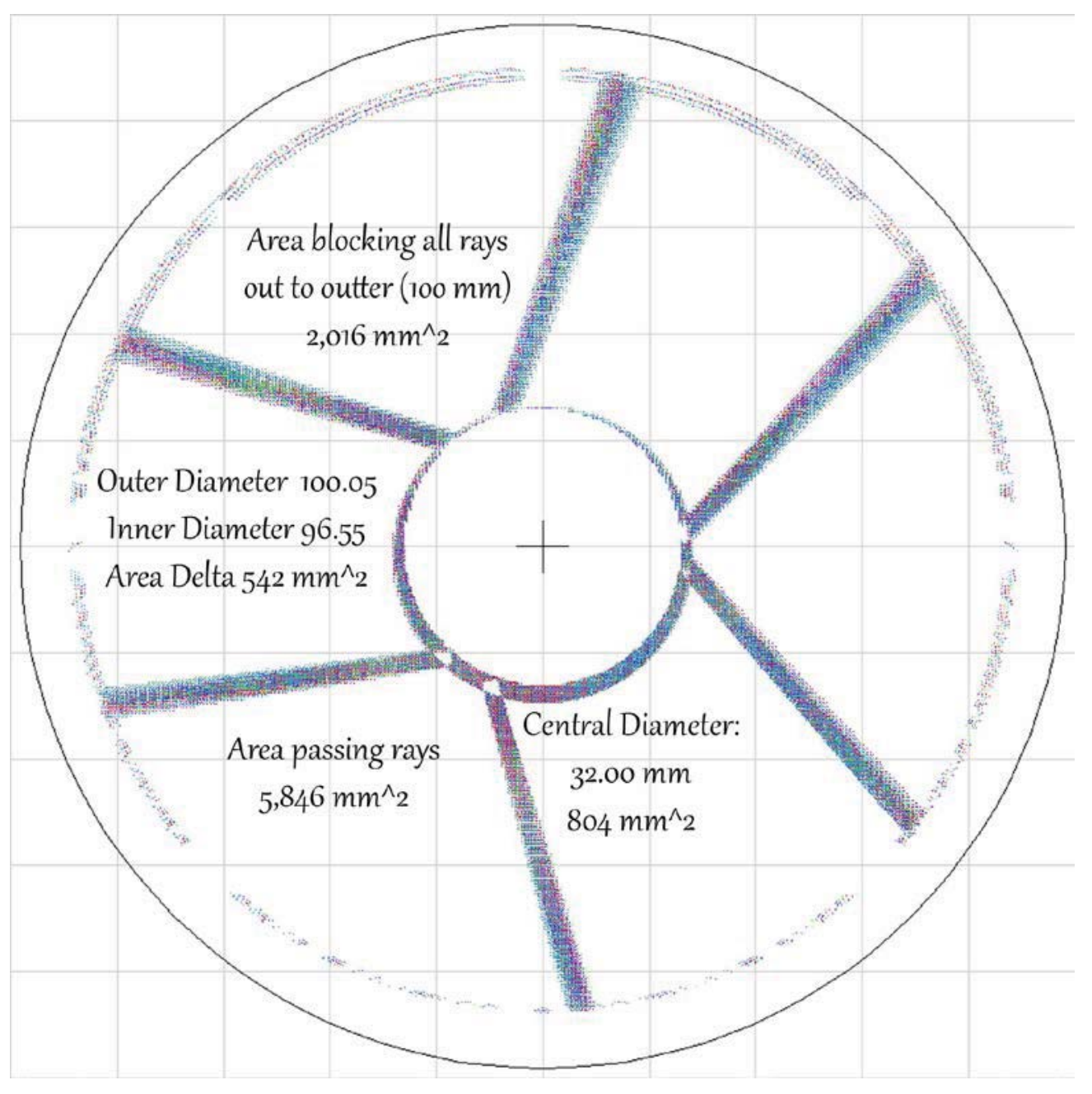

- Average blockage over field:

- Telescope Only:

$15 \%$

- Tel plus Lyot Stop: $23 \%$

- Mask blocks additional $5.1 \%$ to $10.6 \%$ ) 


\section{WIIIR $=\mathbb{I}$ Wide Field Infrared Survey Telescope - AFTA NAgh}

\section{Imager Performance}

- Comparative Polychromatic Spot Sizes

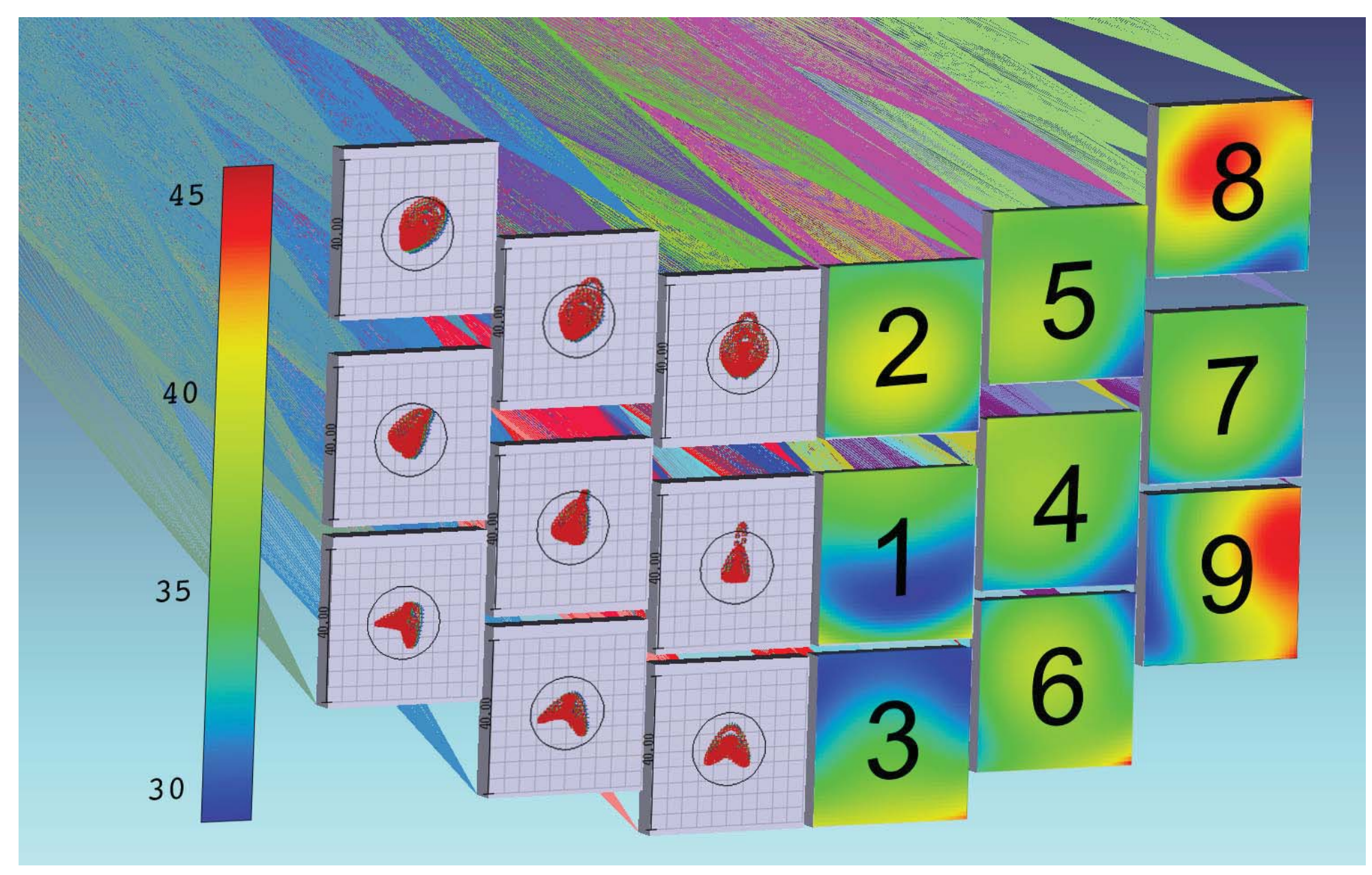




\section{Wide Field Infirared Survey Telescope = AFTA}

\section{WFI Error Budget}

- Pre-Launch Phase:

- Each parameter is budgeted for degrees of freedom in both fabrication and alignment

- Predictions in cooldown and gravity release are accounted for

- These form compensated/uncompensated totals

- All I\&T methods (alignment tools, Wavefront sensing, etc...) and all adjustable (mechanisms, shims, etc.. Are brought to bear for compensation.

- Post-Commissioning

- Errors in analysis and stabilities form new compensated/uncompensated totals

- Only adjustable mechanisms are available for compensators 


\section{W】IDR - TR Wide Field Infrared Survey Telescope - AFTA}

\section{WFI Error Budget (Sample Totals)}

- Error Budget Totals are applied to the Linear Optical Model (LOM)

- Wavefront and boresight changes are calculated.

- Totals compared against system budgets.

- Iterative process, refined as I\&T methods and tools are refined. 


\section{Wide Field Infrared Survey Telescope - AFTA}

\section{Cycle4 WFI design update}

- Outline

- WFI design progress

- Optics performance: distortion, dispersion

- Mechanical, thermal, electrical progress examples

- Error budgeting

- Risk reduction activities

- Cycle5 trade space

- Update on detector development

- Reminder - details of filter set

are pending the telescope
Channel Field Layout for AFTA-WFIRST Instruments

As Projected on Sky

Sunshade

(Payload + Y)

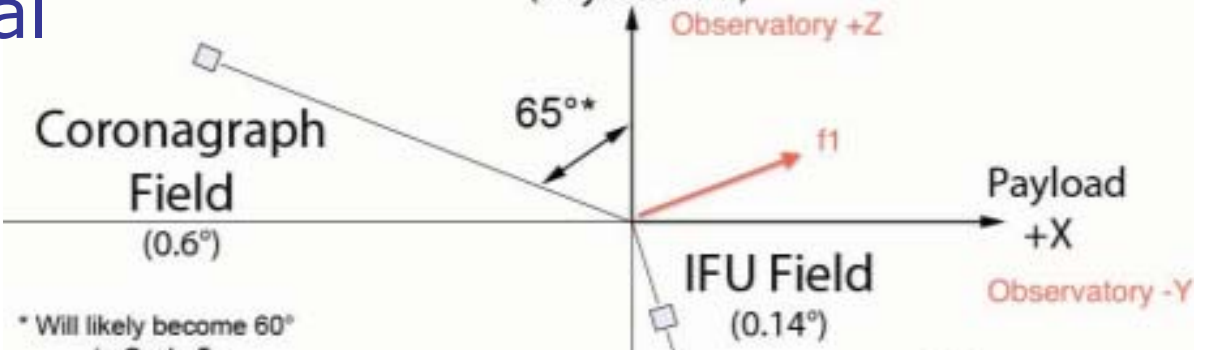

WFI Field (Center $0.617^{\circ}$ )

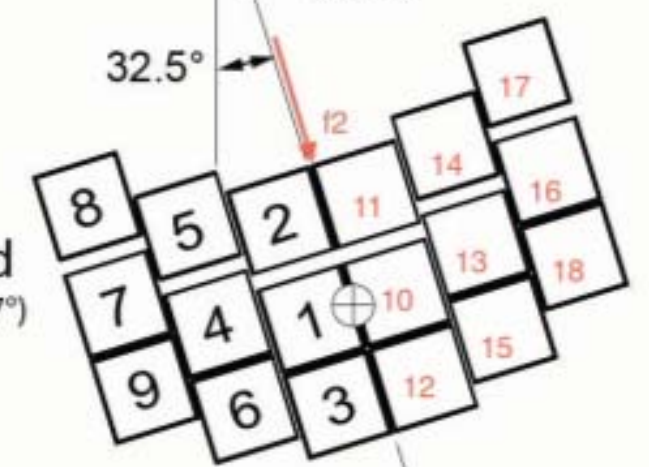

temperature vs risk assessment, ongoing 


\section{Statistical Results4088x4088-p}

- 41x41 sampling of across each sensor

- Each group showed distinct trending:

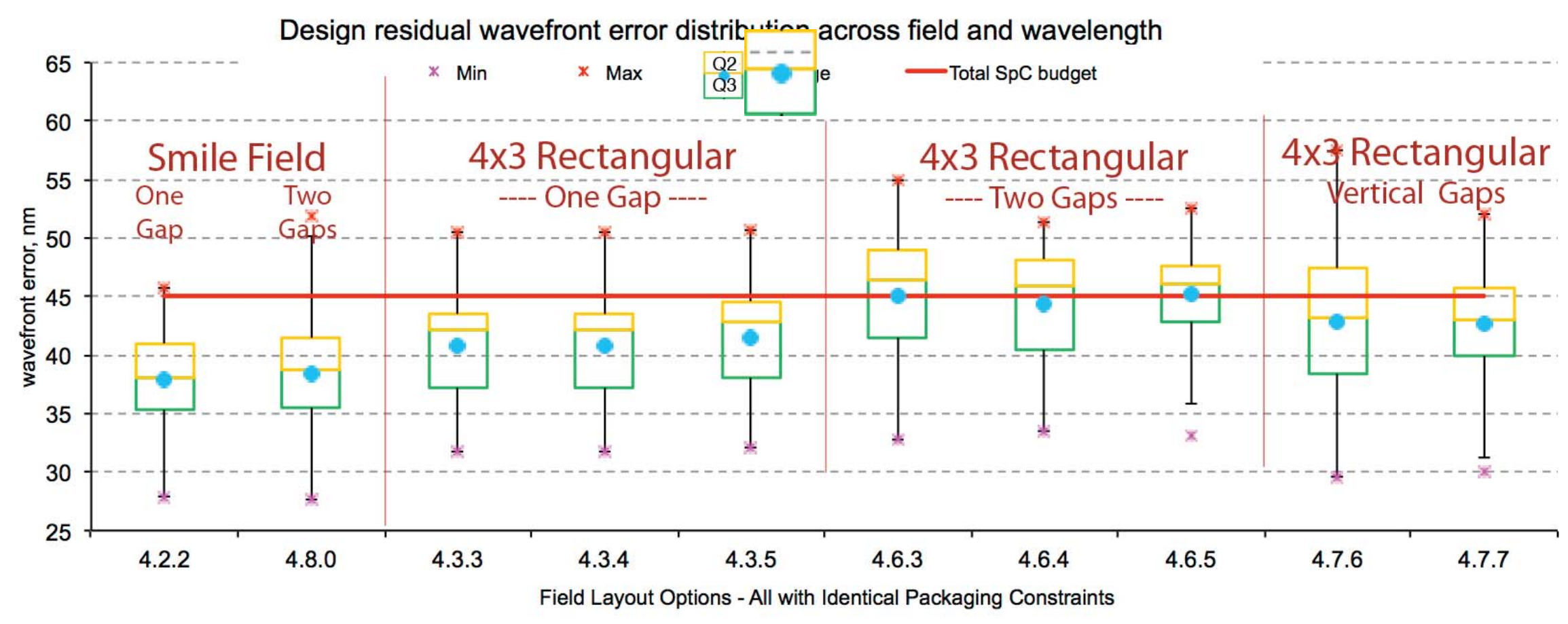




\section{Wide Field Infrared Survey Telescope = AFTA}

\section{Optical distortion}

- Optical distortion assessed

- 'Feature' of TMA optical designs used off field axis

- Independent of the small field configuration tweaks under consideration for Cycle5, see below

- Simple function of radial field angle
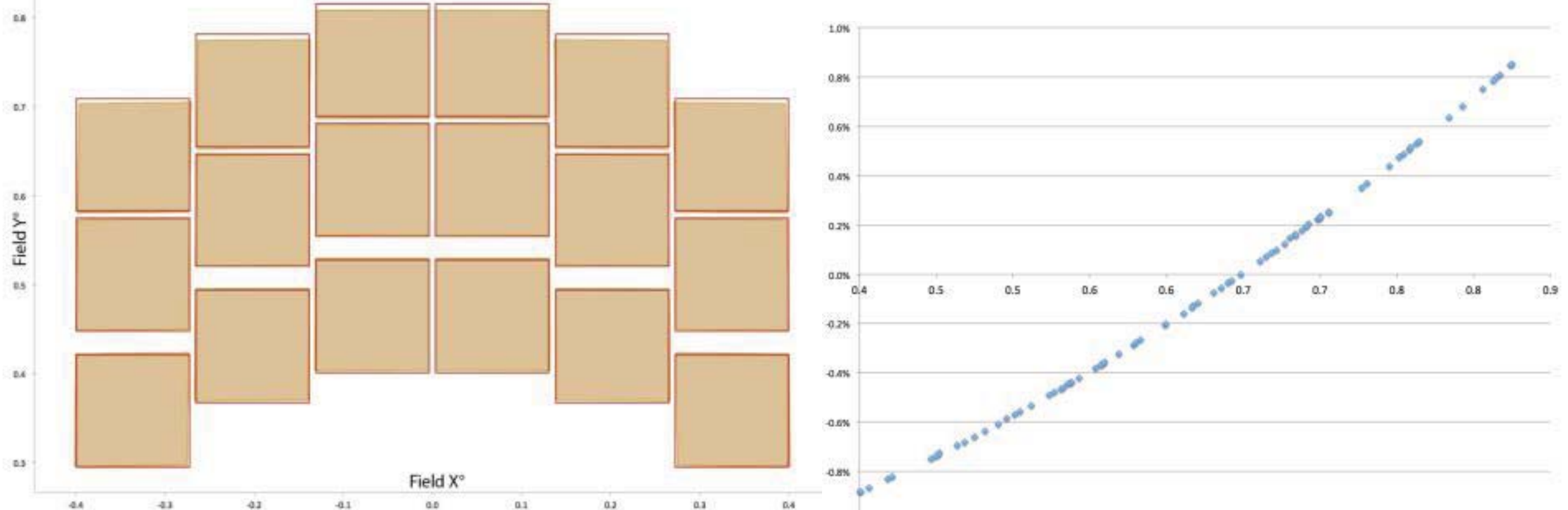

Square outline: zero distortion;

Filled trapezoids: actual SCA field positions

$\mathrm{X}$ : field angle, degrees; Y: Distortion, \% 


\section{Wide Field Infirared Survey Telescope = AFTA}

\section{Grism Layout}

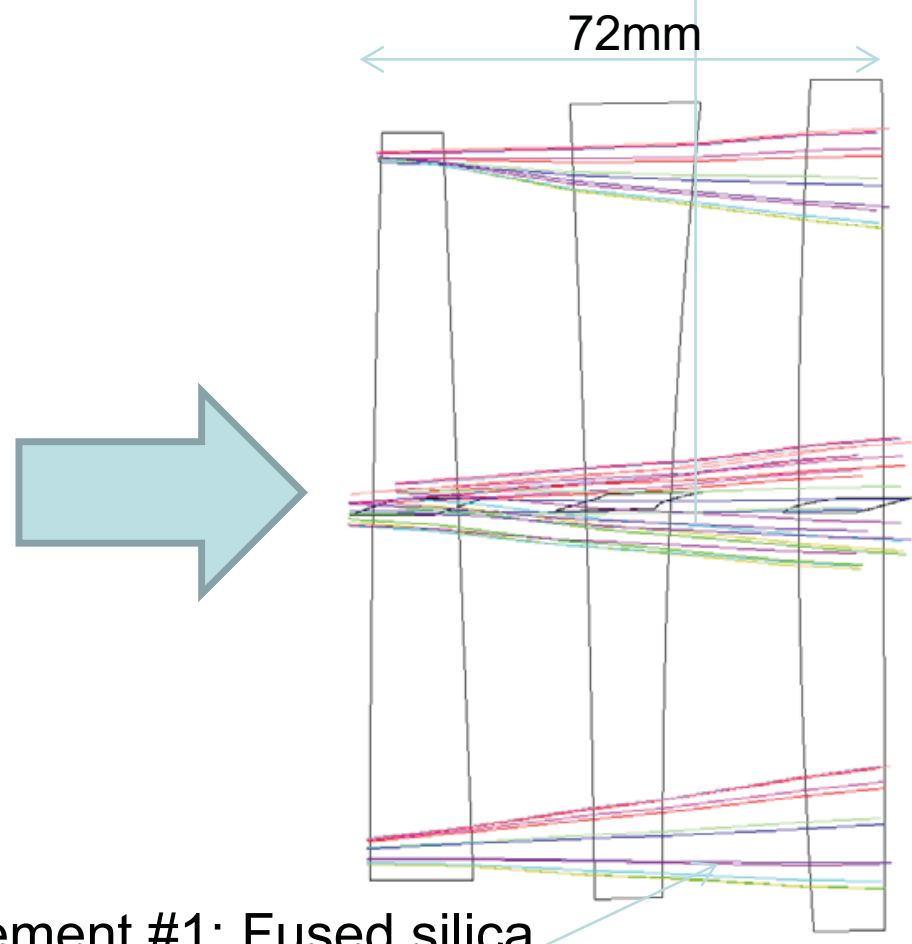

WFIRST/AFTA Grism Element 1 Prototype (Diameter -124mm)

Element \#1: Fused silica Surface \#1: Filter (spherical) Surface \#2: Diffractive lens (flat)

Element \#3: Fused silica Surface \#1: Spherical Surface \#2: Grating (flat)

Element \#2: Fused silica Surface \#1: spherical Surface \#2: Spherical
The filter can be on any spherical surface, but the first surface is smaller, also more perpendicular to the beam. 


\section{Integral Field Channel}

- A separate channel within the Wide-Field instrument enclosure

- Using an assembly of 21 sets of $0.5 \times 10 \mathrm{~mm}$ slicer mirrors, pupil mirrors, and output mirrors, a $3 " \times 3.15^{\prime \prime}$ field is sliced and re-arranged into a continuous slit.

- This slit is then relayed through a spectrograph (R 100), allowing for the multiplexing spectral analysis of each individual $0.15 \times 0.15^{\prime \prime}$ field 


\section{WIIR - TR Wide Field Infrared Survey Telescope - AFTA NASA}

\section{WFIRST IFU Design}

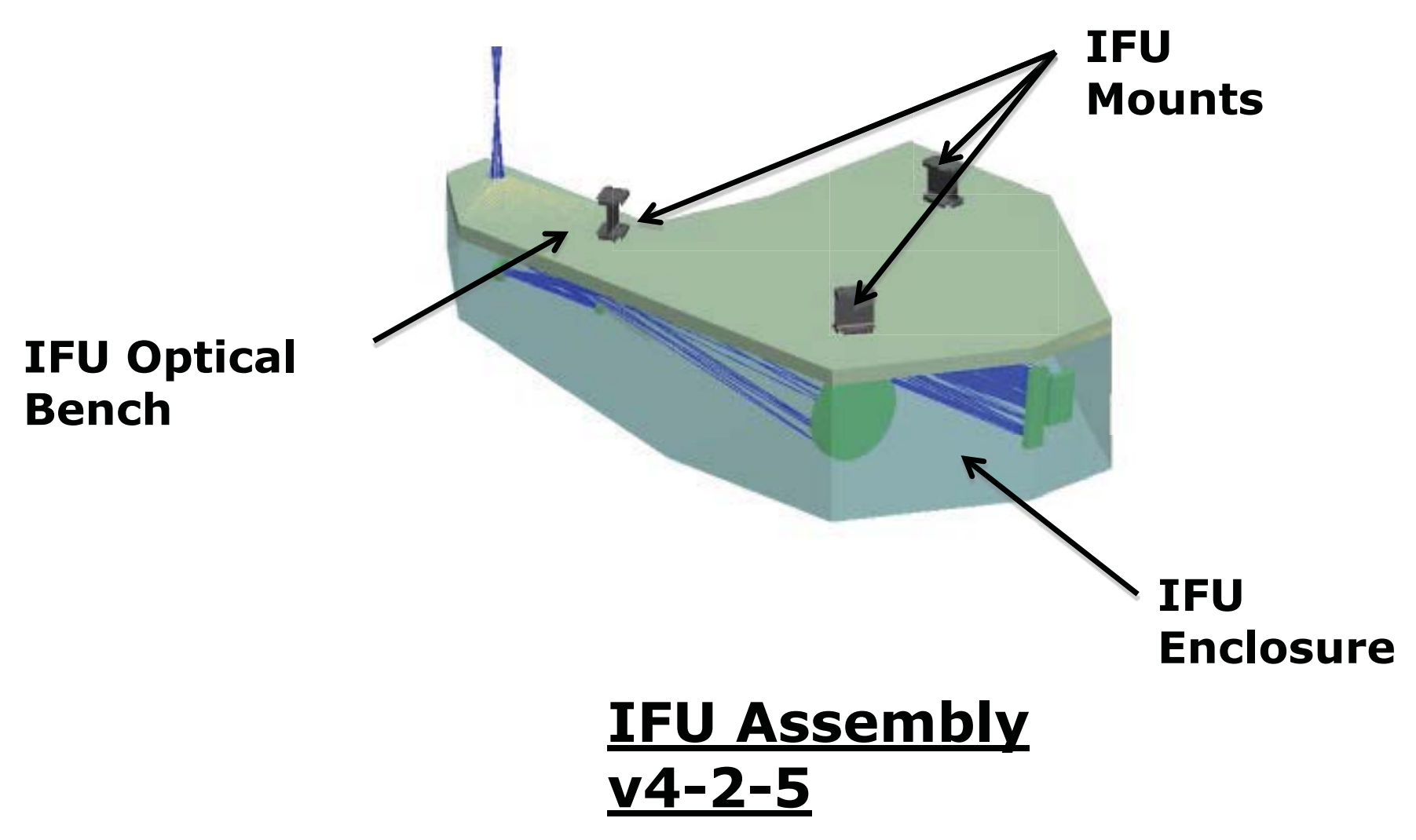




\section{IFU Design Features}

- Uses "current art" for image slicing method

- $2110 \mathrm{~mm} \times 0.5 \mathrm{~mm}$ slicer mirrors form pupils

- 21 pupil mirrors reimage slices to single $50 \mathrm{~mm}$ output slit

- 21 slice output field mirrors create telecentric output

- Spectograph used tri-prism for more constant R

- Collimation and focus mirrors reduce to $18 \mathrm{~mm}$, fits on single H1RG.

- Telecentric input \& output 


\section{WVIFIR $\subset$ TI Wide Field Infrared Survey Telescope - AFTA}

\section{IFU Layout: Slicer with Relay Mirrors}

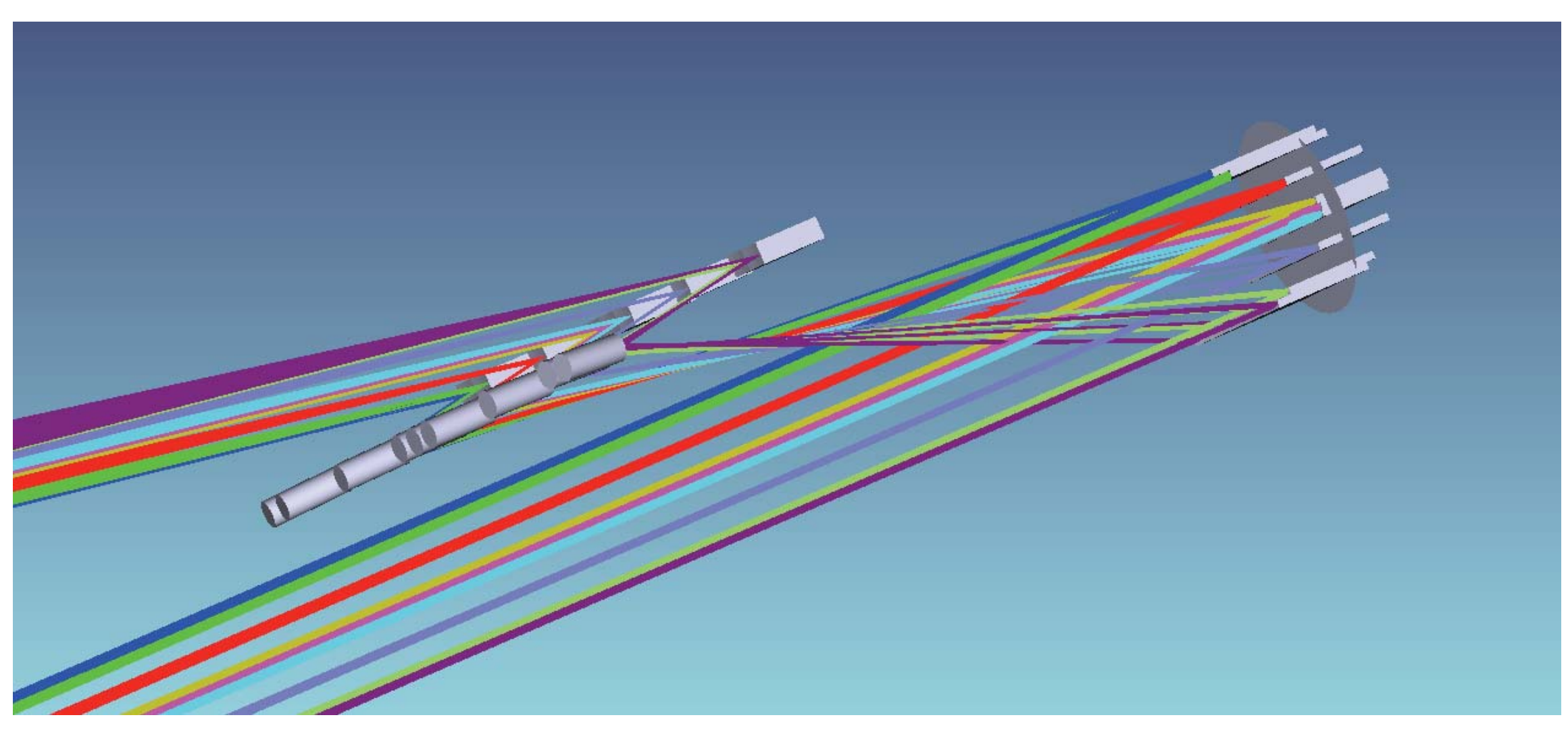




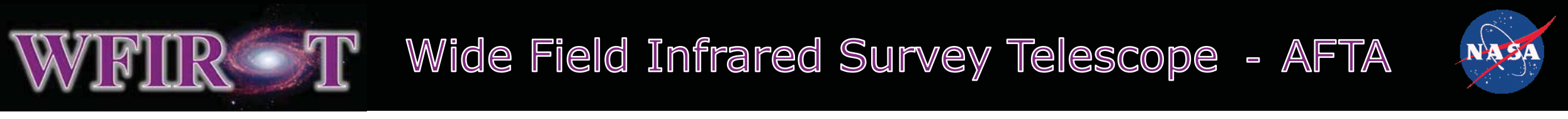

\section{IFU Layout: Slicer with Relay Mirrors}

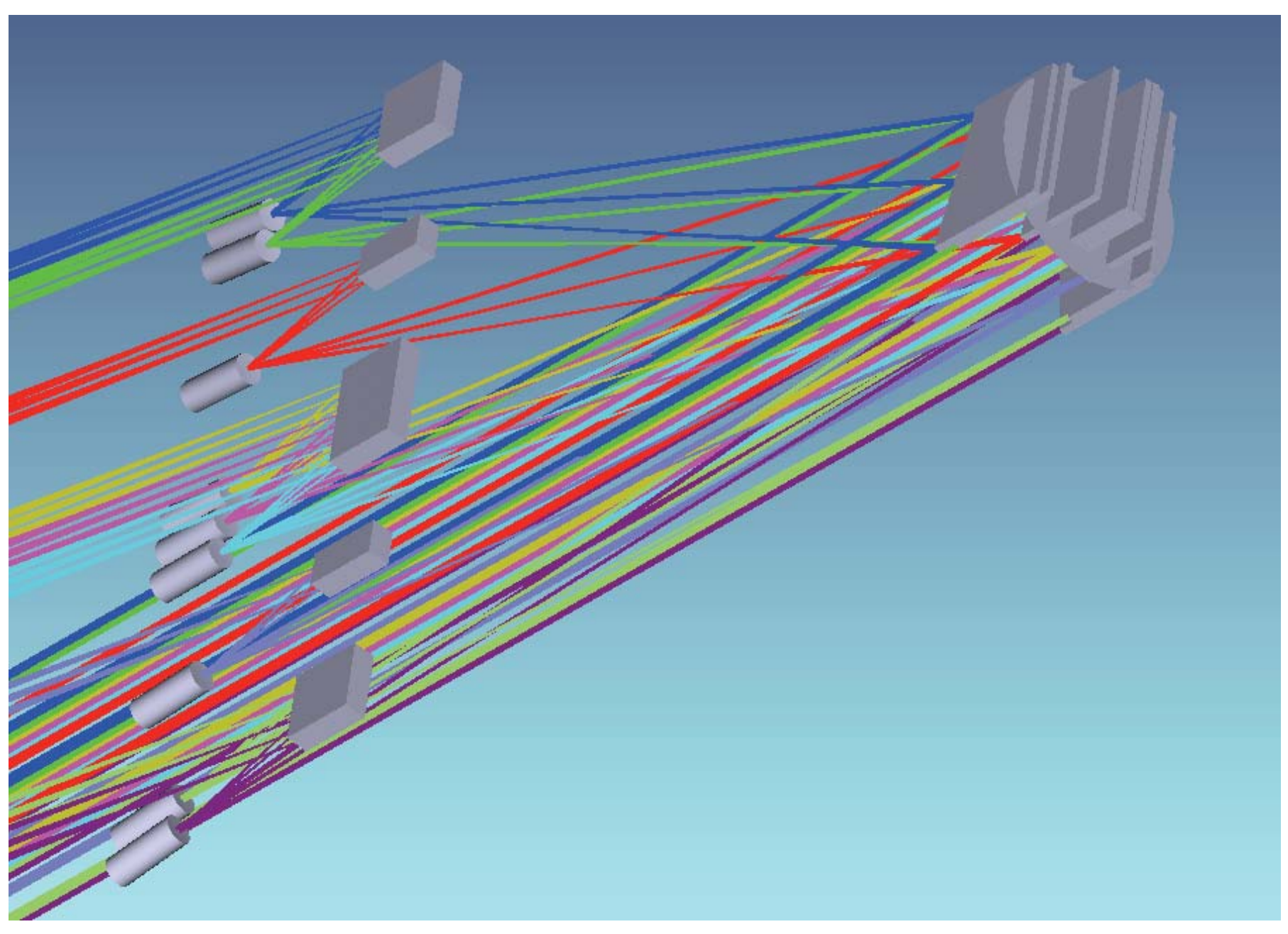




\section{WIIR - TR Wide Field Infrared Survey Telescope - AFTA}

\section{3"x3.15" field cut into $210.5 \mathrm{~mm}$ slices}
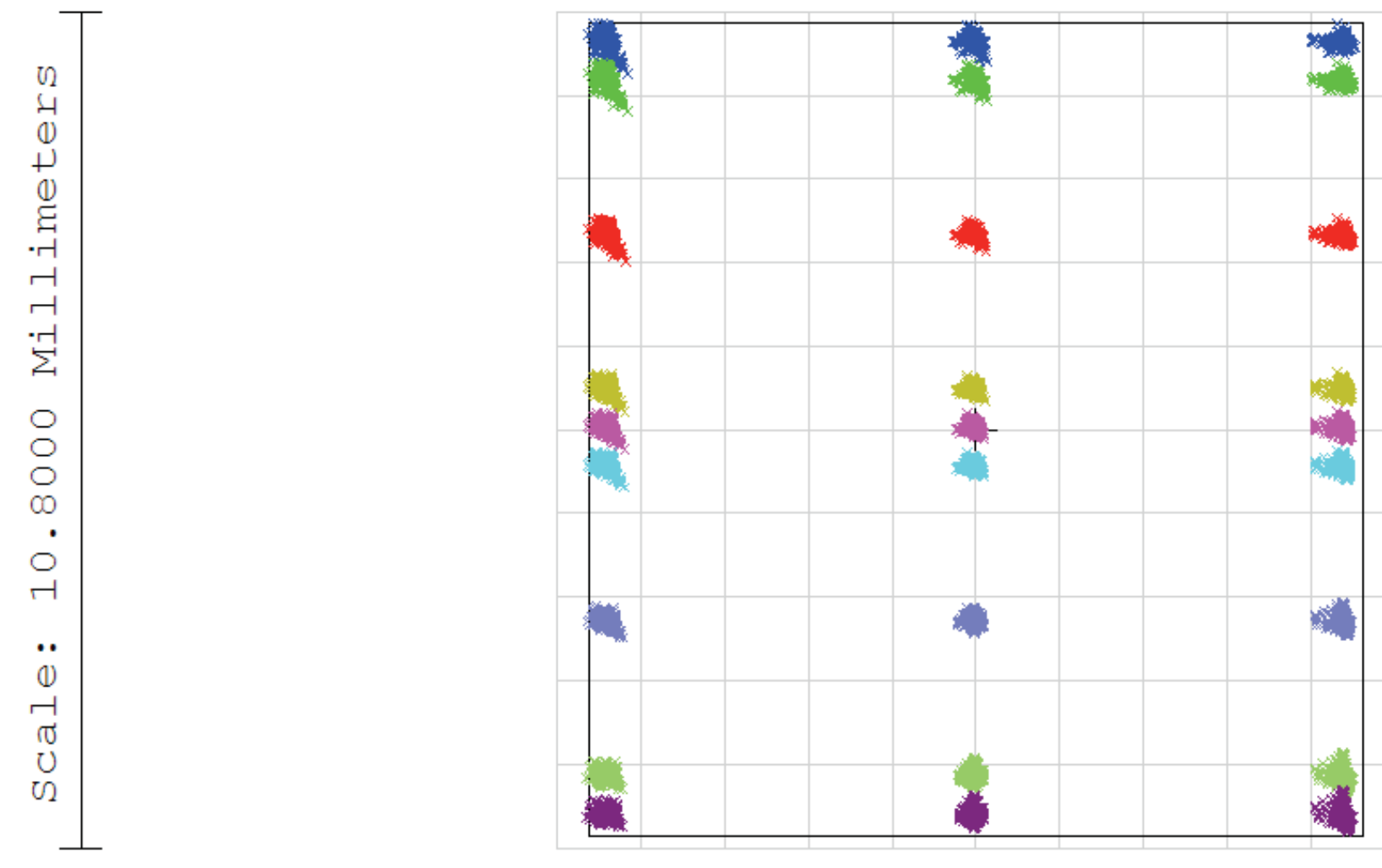


\section{WLIDR - TI Wide Field Infirared Survey Telescope - AFTA NASA}

Final Image Plane: 21 slices dispersed
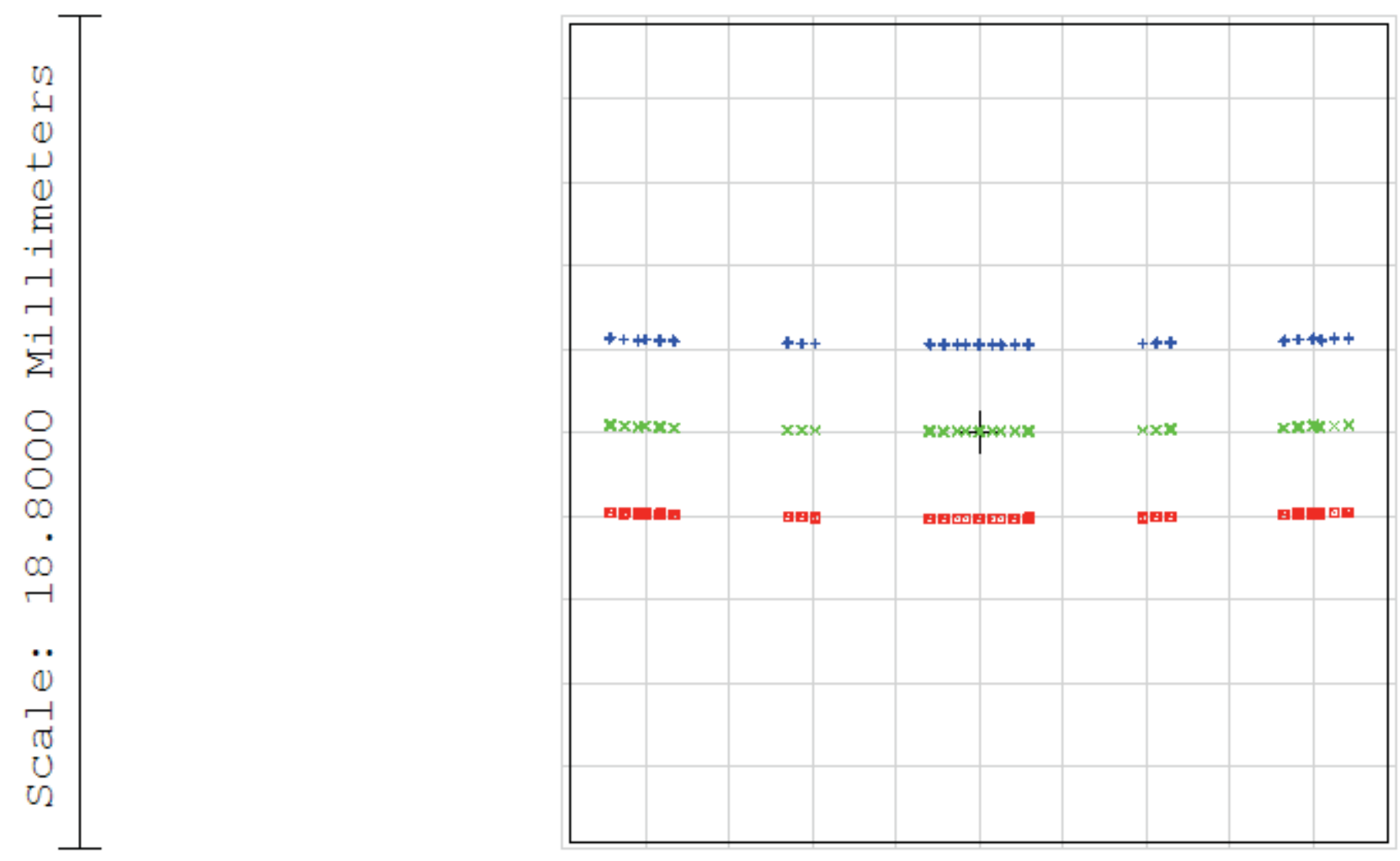


\section{WITIR $=\mathbb{I}$ Wide Field Infrared Survey Telescope - AFTA}

\section{Final Images near diffraction-limited}

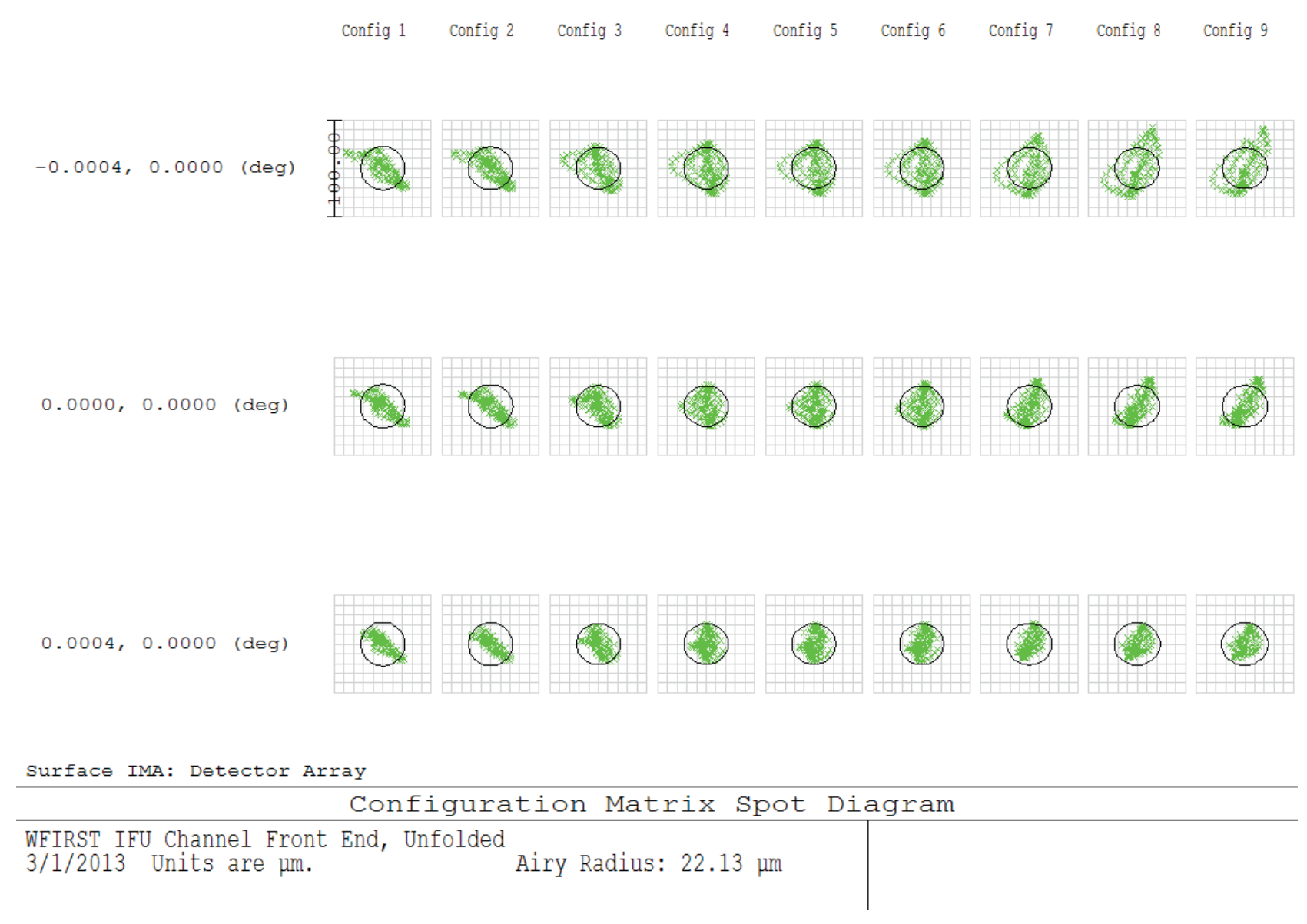




\section{IFU Performance}

- Shortest Wavelength $(0.6 \mu \mathrm{m})$

Config 1

Config 2

Config 3

Config 4

Config 5 Config 6

Config 7

Config 8

Config 9

$-0.0004,0.0000 \mathrm{deg}) \oint_{\Phi_{1}}^{\Phi}$ (4) (4) (40) (40)

$0.0000,0.0000(\mathrm{deg})$

(4) (4) (4) (4) (4) (4) (4)

$0.0004,0.0000(\mathrm{deg})$

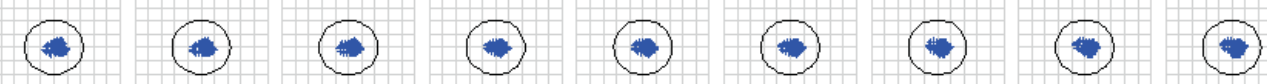

\section{Surface IMA: Detector Array}

Configuration Matrix spot Diagram

\begin{tabular}{|c|c|c|}
\hline $\begin{array}{l}\text { WFIRST Cycle } 4 \text { IFU Relay } \\
9 / 9 / 2013 \text { Units are } \mu \text { m. }\end{array}$ & Airy Radius: $215 \mu \mathrm{m}$ & \\
\hline Scale bar : 1000 & Reference : Chief Ray & $\begin{array}{l}\text { V4-2-1 08-20-13 D. ZMX } \\
\text { Configuration: All } 9\end{array}$ \\
\hline
\end{tabular}




\section{IFU Performance}

- Longest Wavelength $(2 \mu \mathrm{m})$

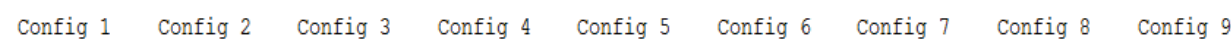

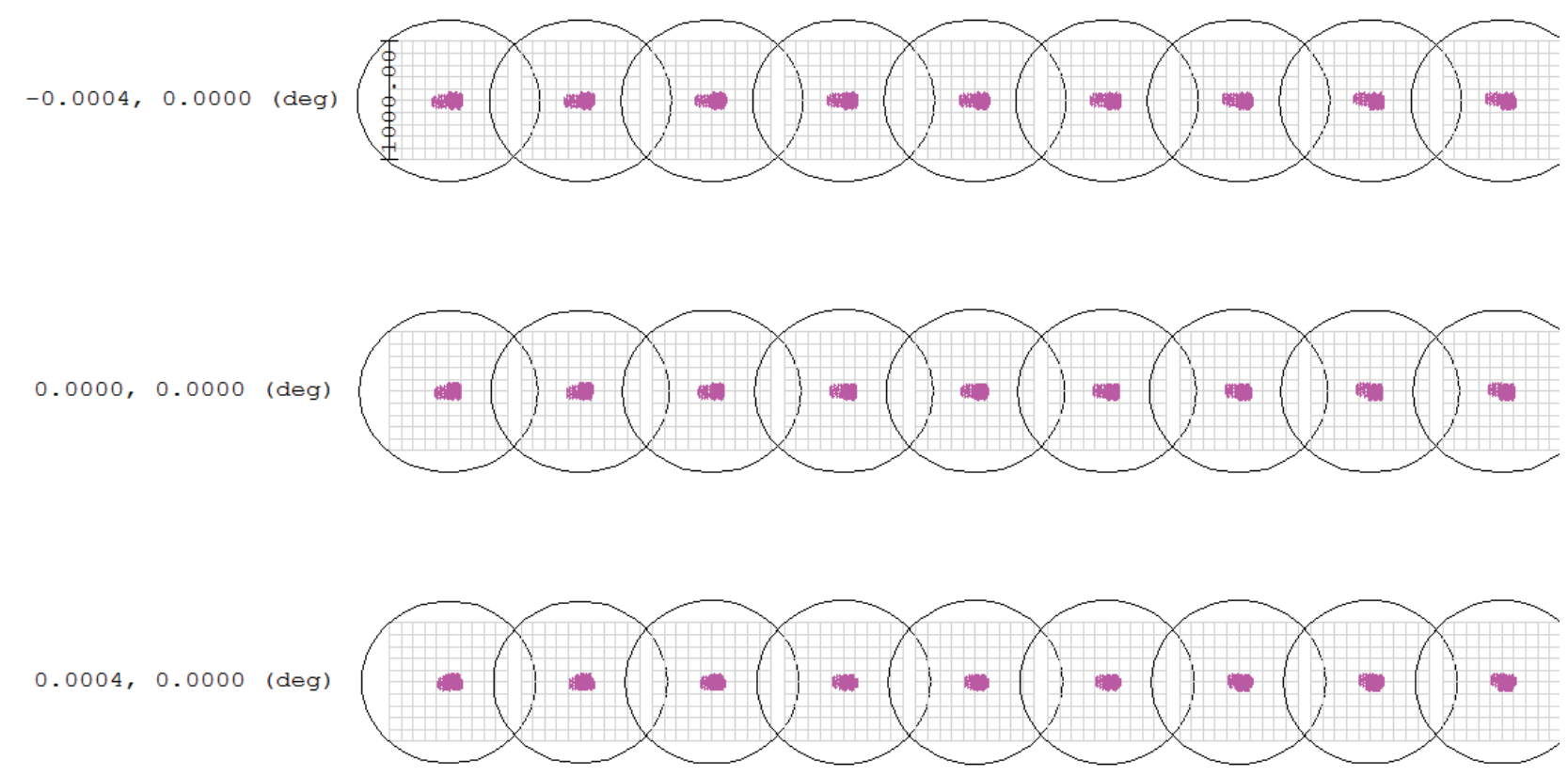

Surface IMA: Detector Array

\begin{tabular}{|c|c|c|}
\hline & Configuration Matrix spot & Diagram \\
\hline $\begin{array}{l}\text { WFIRST Cycle } 4 \text { IFU Relay } \\
9 / 9 / 2013 \text { Units are } \mu m .\end{array}$ & Airy Radius: $716.6 \mu \mathrm{m}$ & \\
\hline Scale bar : 1000 & Reference : Chief Ray & $\begin{array}{l}\text { V4-2-1 08-20-13 D. ZMX } \\
\text { Configuration: All } 9\end{array}$ \\
\hline
\end{tabular}




\section{Wide Field Infrared Survey Telescope - AFTA}

oriay angit mequancincint (eycic zox)

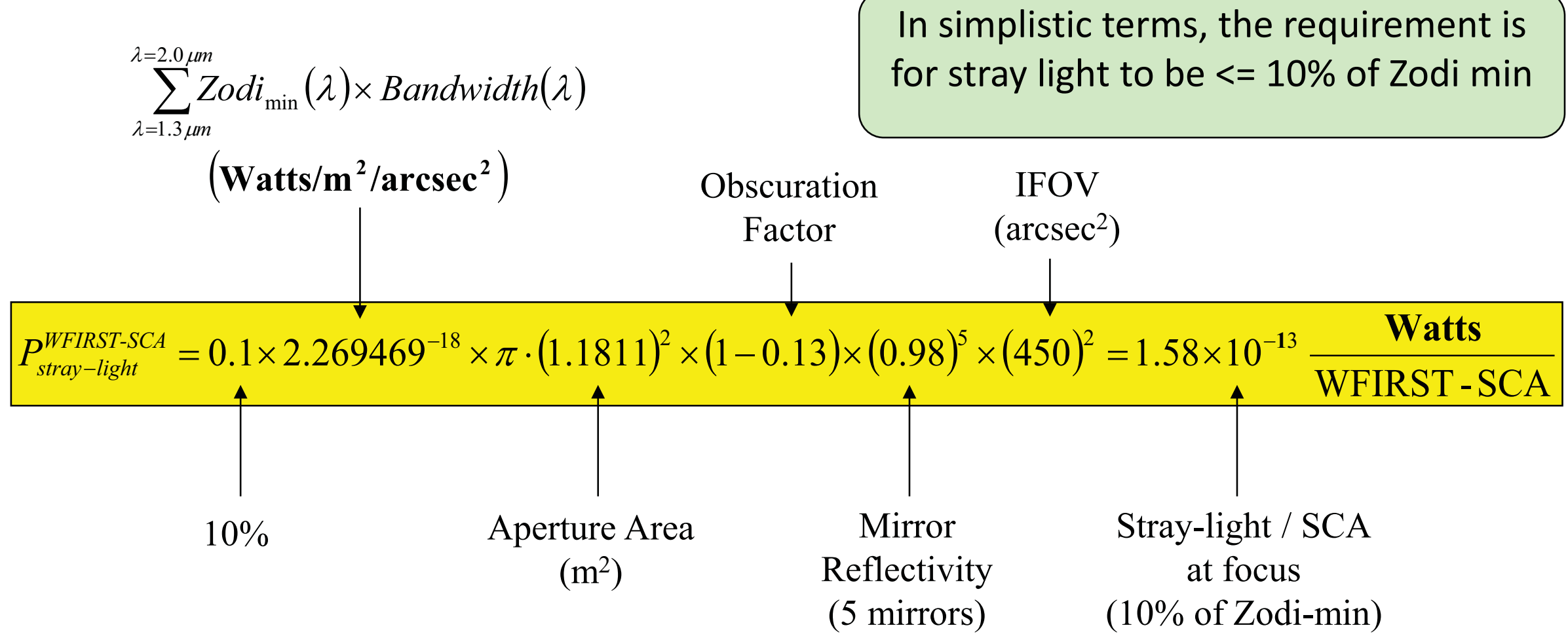

Based on “Cyc1_S-N_Sim_Parameter_Freeze_v5.xls" spreadsheet dated: 1/10/2007

Currently, there is no "official" stray-light requirement for the WFIRST/AFTA, so a tentative "Pass" or "Fail" threshold value of $1.58 \mathrm{e}-13$ Watts/(WFIRST-SCA) is assumed 


\section{Description of PST Calculation (Sunshield)}

Sun, earth and moon are modeled as rising above the horizon of the Sunshield

The values of Irradiance (Power/Area) and Flux (Power) utilized in the irradiance calculations are model and are listed in the appendix

The source is assumed uniform and collimated (far away)

To produce the proceeding graphs the PM aperture is illuminated at various angles

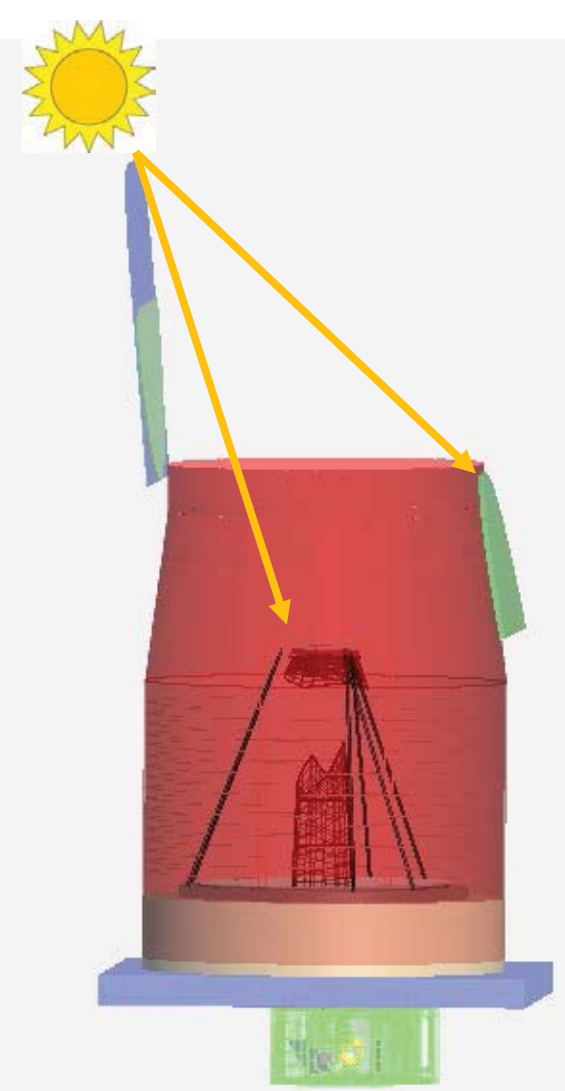

The results are read as detector Irradiance vs. Requirement 


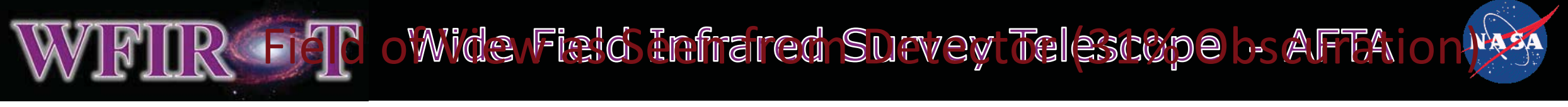

Footprint of Beam on Sky

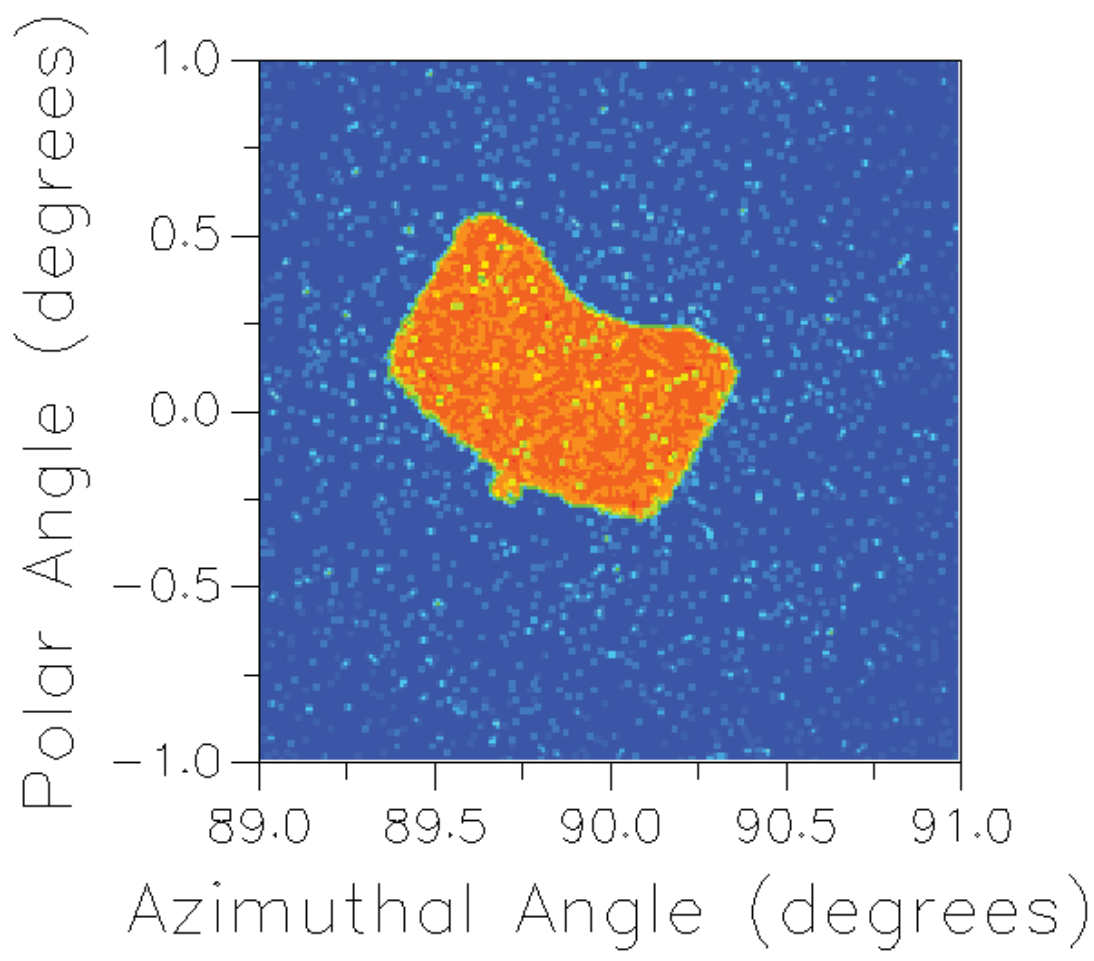

Full Sky View

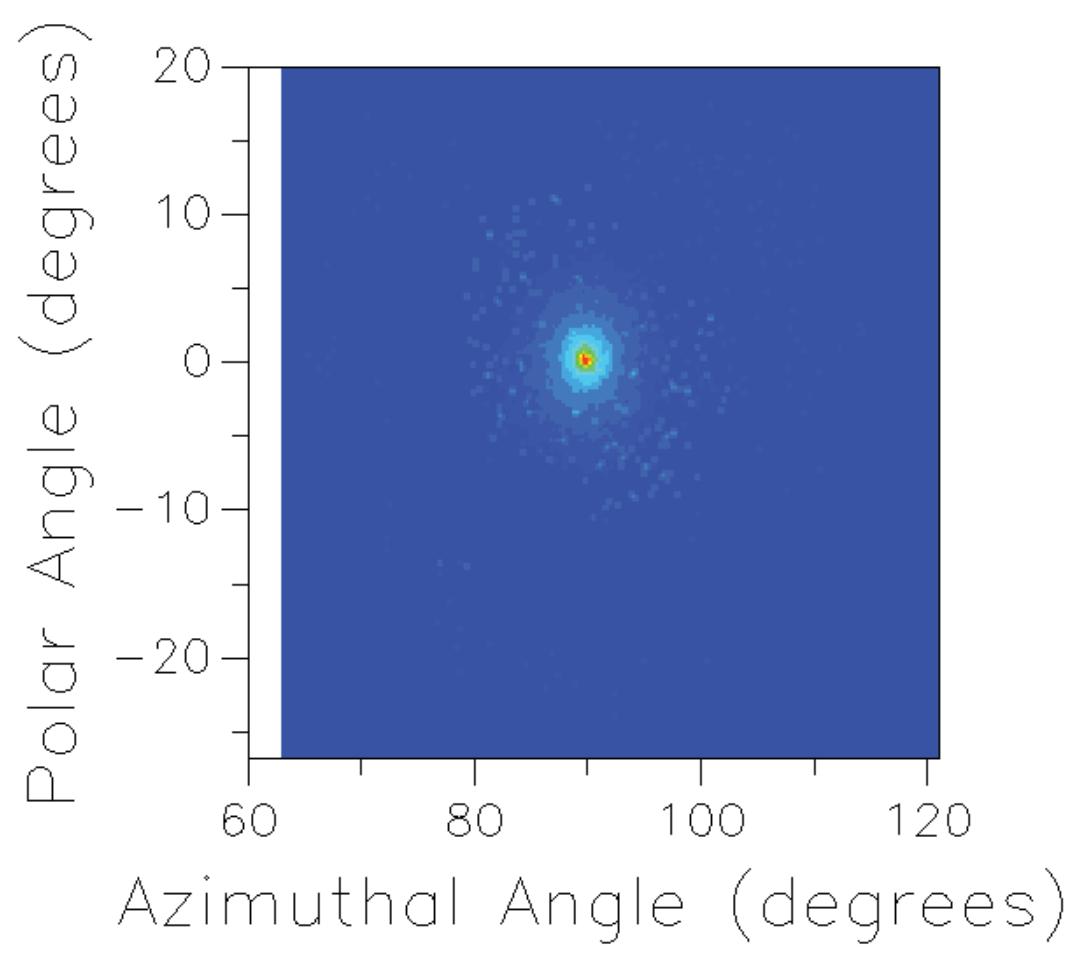


Central Obstruction: Standard Cut vs. Sculptured Baffle (30\% Central Obscuration)

- Rogue (Out-of-field) rays greatly reduced with Sculpted Baffles

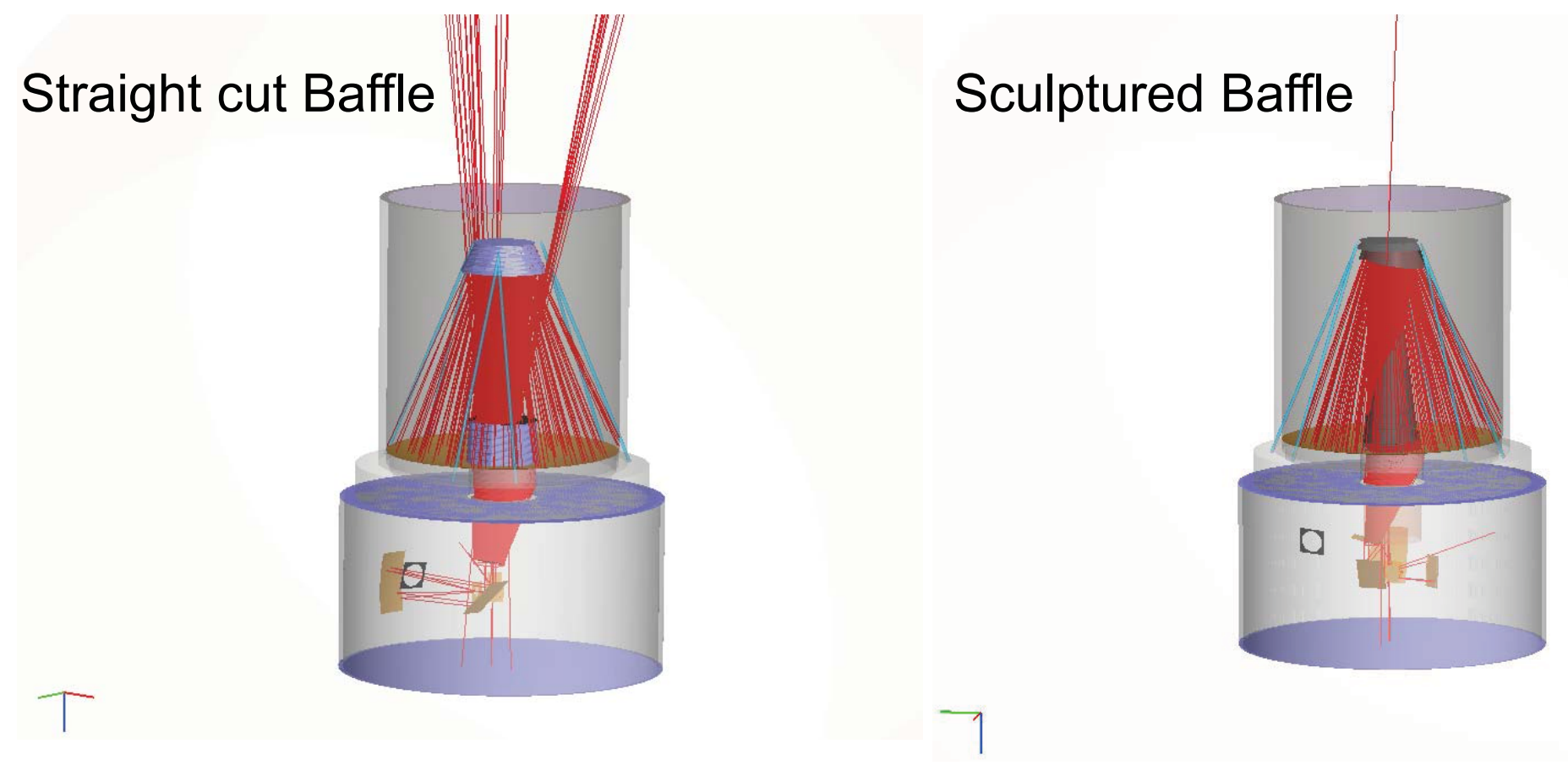




\section{Wide Field Infrared Survey Telescope - AFTA}

\section{Telescope Baffles}

- WFIRST-AFTA's

Telescope baffles will be custom sculpted to match the instrument fields

- Secondary Mirror Shade

- Inner Telescope Baffle

- Primary Mirror edge

- Entrance Aperture Plate

- Reduce Rogue Rays

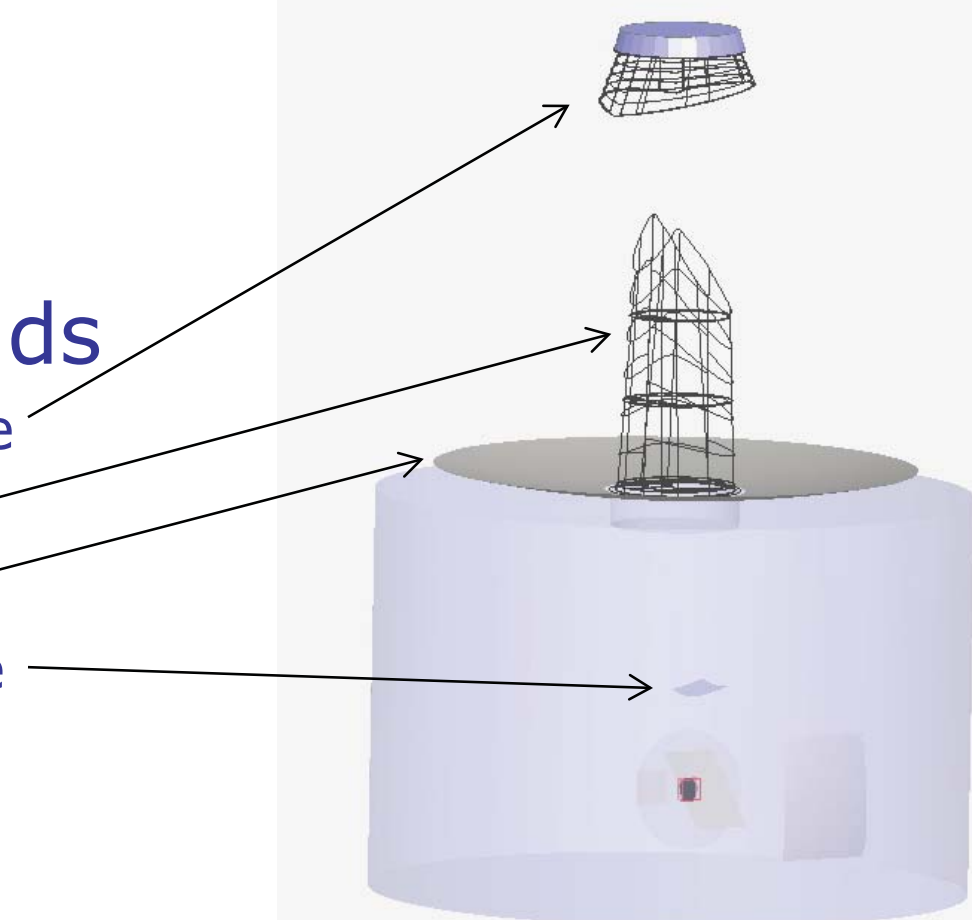

WFIRST-AFTA Stray Light model example 


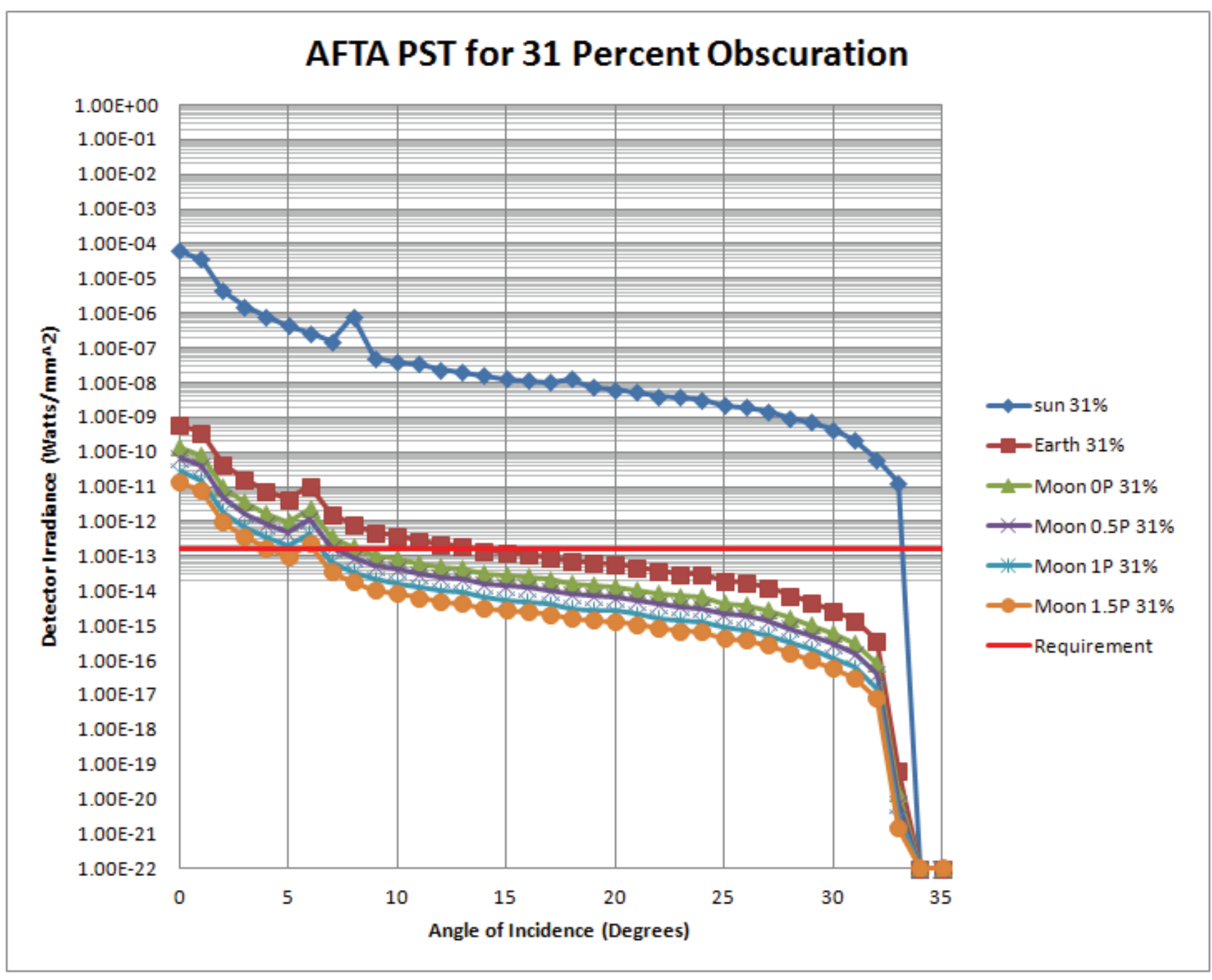




\section{CURRENT EFFORTS}

- Full Bottom-up I\&T error allocation for predicted performance

- Expansion of IFU Field to include 6"x6" 'coarse' $0.3^{\prime \prime}$ sampling field, adjacent to current field

- ... All this in PRE-PHASE-A!

- Launch Date: 2021 - 2024 


\section{For More WFIRST Information:}

- http://wfirst.gsfc.nasa.gov

(or just search "WFIRST" in any search engine)

- Science Definition Team Presentations

- Project Reports

- Contact Project Team Members 